MATHEMATICS OF COMPUTATION

Volume 73 , Number 245 , Pages $35-61$

S $0025-5718(03) 01541-2$

Article electronically published on August 19, 2003

\title{
SUBSTRUCTURING PRECONDITIONERS FOR SADDLE-POINT PROBLEMS ARISING FROM MAXWELL'S EQUATIONS IN THREE DIMENSIONS
}

\author{
QIYA HU AND JUN ZOU
}

\begin{abstract}
This paper is concerned with the saddle-point problems arising from edge element discretizations of Maxwell's equations in a general three dimensional nonconvex polyhedral domain. A new augmented technique is first introduced to transform the problems into equivalent augmented saddlepoint systems so that they can be solved by some existing preconditioned iterative methods. Then some substructuring preconditioners are proposed, with very simple coarse solvers, for the augmented saddle-point systems. With the preconditioners, the condition numbers of the preconditioned systems are nearly optimal; namely, they grow only as the logarithm of the ratio between the subdomain diameter and the finite element mesh size.
\end{abstract}

\section{INTRODUCTION}

In the numerical simulation of electromagnetic models, one needs to repeatedly solve the following system [8, [9], 11], 14], 25], 29], [30]:

$$
\left\{\begin{array}{l}
\operatorname{curl}(\alpha \operatorname{curl} \mathbf{u})+\gamma_{0} \beta \mathbf{u}=\mathbf{f} \quad \text { in } \Omega, \\
\operatorname{div}(\beta \mathbf{u})=g \quad \text { in } \Omega,
\end{array}\right.
$$

with the following boundary condition:

$$
\mathbf{u} \times \mathbf{n}=\mathbf{0} \quad \text { on } \quad \partial \Omega .
$$

Here $\Omega$ is an open, simply connected and Lipschiz domain in $\mathbf{R}^{3}$, and $\mathbf{n}$ is the unit outward normal vector on $\partial \Omega$. The source functions $\mathbf{f} \in L^{2}(\Omega)^{3}$ and $g \in L^{2}(\Omega)$ satisfy the compatibility condition $\gamma_{0} g=\nabla \cdot \mathbf{f}$. The coefficients $\alpha(x)$ and $\beta(x)$ are two positive bounded functions in $\Omega$. In applications, we have $\alpha(x) / \beta(x)=c(x)$ with $c(x)$ being the velocity of light. The constant $\gamma_{0}$ is nonnegative, i.e., $\gamma_{0} \geq 0$, and it is allowed to be identically zero. It is this extreme case that causes the most troublesome technical difficulty to be dealt with in the paper.

Received by the editor February 21, 2002 and, in revised form, July 15, 2002.

2000 Mathematics Subject Classification. Primary 65N30, 65N55.

Key words and phrases. Maxwell's equations, Nédélec finite elements, nonoverlapping domain decomposition, condition numbers.

The work of the first author was supported by Special Funds for Major State Basic Research Projects of China G1999032804.

The work of the second author was partially supported by Hong Kong RGC Grants CUHK4048/02P and CUHK4292/00P. 
For the numerical solution of the system (1.1)-(1.2), the edge finite element methods have been widely used in recent years; see, for example, [8, 9], 21]. It is important to note that the algebraic systems arising from the discretization by the edge element methods are very different from the ones arising from the discretization by the standard nodal finite element methods. Thus the construction of the nonoverlapping domain decomposition preconditioners for the nodal element systems, which has been well developed for the second order elliptic problems in the past two decades (see the survey article [32]), does not work for the edge element discretization of the equations (1.1)-(1.2) in general, especially in three dimensions. Recently, there has been a rapidly growing interest in domain decomposition methods for solving Maxwell's equations. A substructuring domain decomposition method was discussed in [30] for Maxwell's equations in two dimensions, and an overlapping Schwarz method was studied in [13] and 29] for Maxwell's equations in three dimensions. Also, a nonoverlapping domain decomposition method with two subdomains was proposed in [2] for Maxwell's equations in three dimensions. To our knowledge, there exists no work in the literature, which studies nonoverlapping domain decomposition methods for Maxwell's equations in three dimensions for the case with general multiple subdomains. This paper intends to make an initial effort in this direction, and certainly there are still many problems which remain open. As we shall see, for the three-dimensional case with multiple nonoverlapping subdomains, not only the construction of the coarse subspace but also the estimates of the condition numbers of the preconditioned systems are much more difficult and technical than in the two-dimensional case or the three dimensional case with overlapping subdomains.

We will propose an efficient substructuring preconditioner for the saddle-point system arising from the edge element discretizations of the problem (1.1)-(1.2). The most difficult technical issue here lies in the following observation: in the saddlepoint system, the block stiffness matrix corresponding to the operator $\operatorname{curl}(\alpha \operatorname{curl} \cdot)$ for the prime variable $\mathbf{u}$ is singular when $\gamma_{0}=0$ in (1.1); in fact, it is positive semi-definite. How to construct an efficient preconditioner for such saddle-point systems is still an open problem. To overcome this difficulty, we shall first transform the saddle-point system into another equivalent saddle-point problem whose block stiffness matrix corresponding to the prime variable $\mathbf{u}$ is positive definite. The corresponding Schur complement matrix of the saddle-point system can be well preconditioned by some substructuring preconditioners. It will be shown that the resulting preconditioned system has a nearly optimal condition number; namely, it grows only as the logarithm of the ratio between the subdomain diameter and the finite element mesh size.

The outline of the paper is as follows. In Section 2, we describe the edge element discretization of the system (1.1)-(1.2) and introduce some basic formulae and definitions. The construction of nonoverlapping domain decomposition preconditioners and the main results of the paper are discussed in Section 3. Section 4 presents a series of auxiliary lemmata, which will be used to deal with the technical difficulties in the estimates of the condition numbers in Section 5.

\section{EdGe ELEMENT DiscRETiZATION AND DOMAin DECOMPOSition}

This section is devoted to the introduction of the edge element discretization of the system (1.1)-(1.2) and the nonoverlapping domain decomposition. 
2.1. Edge element discretization. The primary goal of this paper is to study the edge element discretization of the equations (1.1)-(1.2) and then to solve the resulting discrete system by a preconditioned iterative Uzawa method with a nonoverlapping domain decomposition preconditioner. First, we shall state the weak formulation of the equations. For this, we need the Sobolev space $H(\operatorname{curl} ; \Omega)$, a space with all square integrable functions whose curl's are also square integrable in $\Omega$. To cope with the boundary condition (1.2), we introduce the following subspaces of $H(\operatorname{curl} ; \Omega)$ :

$$
H_{0}(\operatorname{curl} ; \Omega)=\{\mathbf{v} \in H(\operatorname{curl} ; \Omega) ; \mathbf{v} \times \mathbf{n}=\mathbf{0} \text { on } \partial \Omega\} .
$$

Now, by introducing a Lagrange multiplier $p$ and integration by parts we derive the following variational saddle-point problem associated with the system (1.1)(1.2):

Find $(\mathbf{u}, p) \in H_{0}(\operatorname{curl} ; \Omega) \times H_{0}^{1}(\Omega)$ such that

$$
\left\{\begin{array}{l}
(\alpha \mathbf{c u r l} \mathbf{u}, \mathbf{c u r l} \mathbf{v})+\gamma_{0}(\beta \mathbf{u}, \mathbf{v})+(\nabla p, \beta \mathbf{v})=(\mathbf{f}, \mathbf{v}), \quad \forall \mathbf{v} \in H_{0}(\mathbf{c u r l} ; \Omega) \\
(\beta \mathbf{u}, \nabla q)=(g, q), \quad \forall q \in H_{0}^{1}(\Omega) .
\end{array}\right.
$$

Here and in what follows, $(\cdot, \cdot)$ denotes the scalar product in $L^{2}(\Omega)$ or $L^{2}(\Omega)^{3}$.

Next, we introduce the domain decomposition and the triangulation of the domain $\Omega$, and then we discuss the edge element discretization of the saddle-point problem (2.1).

Domain decomposition. We first decompose $\Omega$ into $N$ nonoverlapping tetrahedral subdomains $\left\{\Omega_{i}\right\}_{i}^{N}$, with each $\Omega_{i}$ of size $d$ (see [4] and 32]). The faces and vertices of the subdomains will be denoted by $\mathrm{F}$ and $\mathrm{V}$, respectively. The common face of the subdomains $\Omega_{i}$ and $\Omega_{j}$ is denoted by $\Gamma_{i j}$. Also, $\Gamma=\bigcup \Gamma_{i j}$, and $\Gamma_{i}=\Gamma \cap \partial \Omega_{i}$. $\Gamma$ will be called the interface. For definiteness, a unique unit normal direction $\mathbf{n}$ is assigned on each face $\mathrm{F}$ of $\Gamma$, and this normal vector is used whenever a unit normal direction is involved on any face in the subsequent analysis.

Finite element triangulation. We further divide each $\Omega_{i}$ into smaller tetrahedral elements of size $h$ so that elements from two neighboring subdomains have an intersection which is either empty or a single nodal point or an edge or a face on the interface $\Gamma$. Let $\mathcal{T}_{h}$ be the resulting triangulation of the domain $\Omega$, which we assume is quasi-uniform. By $\mathcal{E}_{h}$ and $\mathcal{N}_{h}$ we denote the set of edges of $\mathcal{T}_{h}$ and the set of nodes in $\mathcal{T}_{h}$, respectively. Then the Nédélec edge element space, of the lowest order, is a subspace of piecewise linear polynomials defined on $\mathcal{T}_{h}$ (cf. [12] and [22]):

$$
V_{h}(\Omega)=\left\{\mathbf{v} \in H_{0}(\operatorname{curl} ; \Omega) ;\left.\mathbf{v}\right|_{K} \in R(K), \forall K \in \mathcal{T}_{h}\right\}
$$

where $R(K)$ is a subset of all linear polynomials on the element $K$ of the form:

$$
R(K)=\left\{\mathbf{a}+\mathbf{b} \times \mathbf{x} ; \mathbf{a}, \mathbf{b} \in \mathbf{R}^{3}, \mathbf{x} \in K\right\} .
$$

It is well known that for any $\mathbf{v} \in V_{h}(\Omega)$, its tangential components are continuous on all edges of each element in the triangulation $\mathcal{T}_{h}$. Moreover, each edge element function $\mathbf{v}$ in $V_{h}(\Omega)$ is uniquely determined by its moments on each edge $e$ of $\mathcal{T}_{h}$ :

$$
\left\{\lambda_{e}(\mathbf{v})=\int_{e} \mathbf{v} \cdot \mathbf{t}_{e} d s ; e \in \mathcal{E}_{h}\right\}
$$

where $\mathbf{t}_{e}$ denotes the unit vector on the edge $e$. 
Let $\left\{L_{e} ; e \in \mathcal{E}_{h}\right\}$ be the edge element basis functions of $V_{h}(\Omega)$ satisfying

$$
\lambda_{e^{\prime}}\left(L_{e}\right)= \begin{cases}1, & \text { if } e^{\prime}=e, \\ 0, & \text { if } e^{\prime} \neq e .\end{cases}
$$

Then each function $\mathbf{v}$ in $V_{h}(\Omega)$ can be expressed as

$$
\mathbf{v}(\mathbf{x})=\sum_{e \in \mathcal{E}_{h}} \lambda_{e}(\mathbf{v}) L_{e}(\mathbf{x}), \quad \mathbf{x} \in \Omega .
$$

By $Z_{h}(\Omega)$ we denote the continuous piecewise linear finite element subspace of $H_{0}^{1}(\Omega)$ associated with the triangulation $\mathcal{T}_{h}$. Then the saddle-point system (2.1) may be approximated by the finite element problem: Find $\left(\mathbf{u}_{h}, p_{h}\right) \in V_{h}(\Omega) \times Z_{h}(\Omega)$ such that

$$
\left\{\begin{array}{l}
\left(\alpha \operatorname{curl} \mathbf{u}_{h}, \operatorname{curl} \mathbf{v}_{h}\right)+\gamma_{0}\left(\beta \mathbf{u}_{h}, \mathbf{v}_{h}\right)+\left(\nabla p_{h}, \beta \mathbf{v}_{h}\right)=\left(\mathbf{f}, \mathbf{v}_{h}\right), \quad \forall \mathbf{v}_{h} \in V_{h}(\Omega), \\
\left(\beta \mathbf{u}_{h}, \nabla q_{h}\right)=\left(g, q_{h}\right), \quad \forall q_{h} \in Z_{h}(\Omega) .
\end{array}\right.
$$

2.2. Some edge element spaces and discrete operators. Before formulating the domain decomposition preconditioner to be used for solving the system (2.2), we introduce some useful notations and discrete operators.

Let $G$ be either the entire interface $\Gamma$ or the local interface $\Gamma_{i}$ or a face $\mathrm{F}$ of $\Gamma_{i}$. We shall frequently use the restrictions of the tangential components of the functions in $V_{h}(\Omega)$ on $G$ :

$$
V_{h}(G)=\left\{\boldsymbol{\psi} \in L^{2}(G)^{3} ; \boldsymbol{\psi}=\mathbf{v} \times \mathbf{n} \text { on } G \text { for some } \mathbf{v} \in V_{h}(\Omega)\right\} .
$$

The restrictions of $V_{h}(\Omega)$ on each subdomain $\Omega_{i}$ is denoted by $V_{h}\left(\Omega_{i}\right)$. The following local subspaces of $V_{h}\left(\Omega_{i}\right)$ and $V_{h}(\mathrm{~F})$ will be important to our analysis:

$$
\begin{aligned}
V_{h}^{0}\left(\Omega_{i}\right) & =\left\{\mathbf{v} \in V_{h}\left(\Omega_{i}\right) ; \mathbf{v} \times \mathbf{n}=0 \text { on } \Gamma_{i}\right\}, \\
V_{h}^{0}(\mathrm{~F}) & =\left\{\Phi=\mathbf{v} \times \mathbf{n} \in V_{h}(\mathrm{~F}) ; \lambda_{e}(\mathbf{v})=0, \forall e \subset \partial \mathrm{F} \cap \mathcal{E}_{h}\right\} .
\end{aligned}
$$

The natural restriction operator from $V_{h}(\Gamma)$ onto $V_{h}(G)$ and the natural zero extension operator from $V_{h}(G)$ into $L^{2}(\Gamma)^{3}$ will be denoted as $\mathbf{I}_{G}$ and $\mathbf{I}_{G}^{t}$, respectively. For a face F, it is easy to see that $\mathbf{I}_{\mathrm{F}}^{t} \mathbf{v} \in V_{h}(\Gamma)$ if and only if $\mathbf{v} \in V_{h}^{0}(\mathrm{~F})$, and $\mathbf{I}_{G}$ and $\mathbf{I}_{G}^{t}$ satisfy

$$
\left\langle\mathbf{I}_{G} \Psi, \Phi\right\rangle_{G}=\left\langle\Psi, \mathbf{I}_{G}^{t} \Phi\right\rangle \quad \forall \Psi \in V_{h}(\Gamma), \Phi \in V_{h}(G) .
$$

Here and hereafter, $\langle\cdot, \cdot\rangle_{G}$ stands for the $L^{2}$-inner product in $L^{2}(G)$ or $L^{2}(G)^{3}$, and we will drop the subscript $G$ when $G=\Gamma$. For simplicity, we shall often write $\mathbf{I}_{i j}^{t}=\mathbf{I}_{\Gamma_{i j}}^{t}$.

For any face $\mathrm{F}$ of $\Omega_{i}$, we use $\mathrm{F}_{b}$ to denote the union of all $\mathcal{T}_{h}$-induced (closed) triangles on $\mathrm{F}$, which have either one single vertex or one edge lying on $\partial \mathrm{F}$, and $\mathrm{F}_{\partial}$ to denote the open set $\mathrm{F} \backslash \mathrm{F}_{b}$; see Figure 1 . For any subdomain $\Omega_{i}$, define

$$
\Delta_{i}=\bigcup_{\mathrm{F} \subset \Gamma_{i}} \mathrm{~F}_{b}, \quad i=1, \cdots, N .
$$

From now on, the notation $e$, with $e \subset G \subset \Gamma_{i}$, always means that $e$ is an edge of $\mathcal{T}_{h}$ and lies on $G$. 


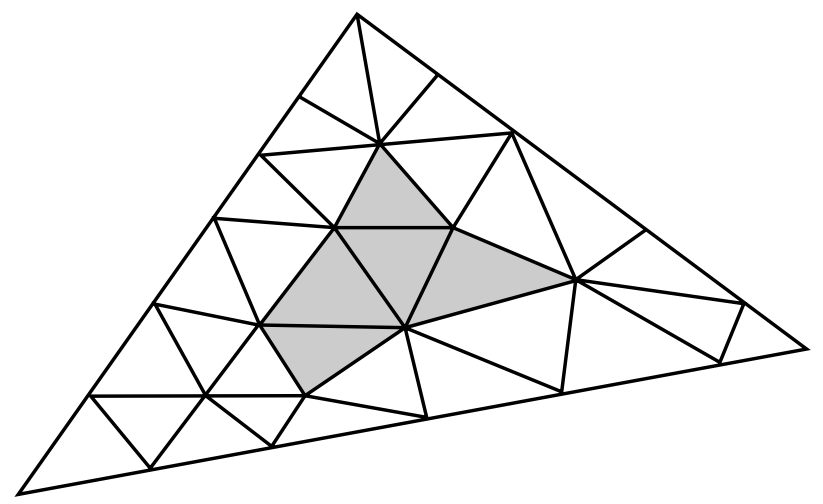

Figure 1. Face $\mathrm{F}$, its boundary subset $\mathrm{F}_{b}$ (union of white triangles) and its interior subset $\mathrm{F}_{\partial}$ (union of shaded triangles)

By definition, for any $\Phi \in V_{h}\left(\Gamma_{i}\right)$, there exists a $\mathbf{v} \in V_{h}\left(\Omega_{i}\right)$ such that $\Phi=\mathbf{v} \times \mathbf{n}$ on $\Gamma_{i}$. Thus we can write

$$
\Phi(\mathbf{x})=\sum_{e \subset \Gamma_{i}} \lambda_{e}(\mathbf{v})\left(L_{e} \times \mathbf{n}\right)(\mathbf{x}), \quad \mathbf{x} \in \Gamma_{i} .
$$

Furthermore, for an open face $\mathrm{F} \subset \Gamma_{i}$, we define an operator $\mathbf{I}_{\mathrm{F} \partial}^{0}: V_{h}\left(\Gamma_{i}\right) \rightarrow$ $\mathbf{I}_{\mathrm{F}}^{t} V_{h}^{0}(\mathrm{~F})$ by

$$
\left(\mathbf{I}_{\mathrm{F}_{\partial}}^{0} \Phi\right)(\mathbf{x})=\sum_{e \subset \mathrm{F}_{\partial}} \lambda_{e}(\mathbf{v})\left(L_{e} \times \mathbf{n}\right)(\mathbf{x}), \quad \mathbf{x} \in \Gamma_{i},
$$

and an operator $\mathbf{I}_{\mathrm{F}_{b}}^{0}$ by

$$
\left(\mathbf{I}_{\mathrm{F}_{b}}^{0} \Phi\right)(\mathbf{x})=\sum_{e \subset \mathrm{F}_{b}} \lambda_{e}(\mathbf{v}) \mathbf{I}_{\mathrm{F}}^{t}\left(L_{e} \times \mathbf{n}\right)(\mathbf{x}), \quad \mathbf{x} \in \Gamma_{i} .
$$

Similarly, we define for any $\mathrm{x} \in \Gamma_{i}$,

$$
\mathbf{I}_{\mathrm{F}}^{0} \Phi(\mathbf{x})=\sum_{e \subset \mathrm{F}} \lambda_{e}(\mathbf{v}) \mathbf{I}_{\mathrm{F}}^{t}\left(L_{e} \times \mathbf{n}\right)(\mathbf{x}) ; \quad \mathbf{I}_{\Delta_{i}}^{0} \Phi(\mathbf{x})=\sum_{e \subset \Delta_{i}} \lambda_{e}(\mathbf{v})\left(L_{e} \times \mathbf{n}\right)(\mathbf{x}) .
$$

Though our main focus in this paper is on the edge element spaces, we shall also make use of some nodal element spaces in the subsequent analyses. As defined earlier, $Z_{h}(\Omega)$ is the continuous piecewise linear finite element space of $H_{0}^{1}(\Omega)$ associated with $\mathcal{T}_{h}$. The restrictions of $Z_{h}(\Omega)$ on $\Gamma$ and $\Gamma_{i}$, in each subdomain $\Omega_{i}$ and on each face $\mathrm{F}$ will be denoted by $Z_{h}(\Gamma), Z_{h}\left(\Gamma_{i}\right), Z_{h}\left(\Omega_{i}\right)$ and $Z_{h}(\mathrm{~F})$, respectively. The operator $\mathrm{I}_{\mathrm{F}}^{t}: Z_{h}(\mathrm{~F}) \rightarrow L^{2}(\Gamma)$ denotes the natural zero extension from $\mathrm{F}$ onto $\Gamma$.

For a subset $G$ of $\Gamma_{i}$, we define a "local" subspace

$$
Z_{h}^{0}(G)=\left\{v \in Z_{h}\left(\Gamma_{i}\right) ; v=0 \text { at all nodes on } \Gamma_{i} \backslash G\right\} .
$$

For any open face $\mathrm{F} \subset \Gamma_{i}$, the operators $\mathrm{I}_{\mathrm{F}}^{0}: Z_{h}\left(\Gamma_{i}\right) \rightarrow Z_{h}^{0}(\mathrm{~F})$ and $\mathrm{I}_{\partial \mathrm{F}}^{0}: Z_{h}\left(\Gamma_{i}\right) \rightarrow$ $Z_{h}^{0}(\partial \mathrm{F})$ denote the following restriction operators:

$$
\mathrm{I}_{G}^{0} w_{h}\left(x_{i}\right)=\left\{\begin{array}{l}
w_{h}\left(x_{i}\right), \quad x_{i} \in G \cap \mathcal{N}_{h} \\
0, \quad x_{i} \in\left(\Gamma_{i} \backslash G\right) \cap \mathcal{N}_{h}
\end{array} \quad \text { for } G=\mathrm{F} \text { or } \partial \mathrm{F} \text { and any } w_{h} \in Z_{h}\left(\Gamma_{i}\right) .\right.
$$


We end this section with the introduction of two frequently used extension operators. With each subdomain $\Omega_{i}$, we define the local operator $A_{i}: V_{h}\left(\Omega_{i}\right) \rightarrow V_{h}\left(\Omega_{i}\right)$ by

$$
\left(A_{i} \mathbf{u}, \mathbf{v}\right)=(\alpha \mathbf{c u r l} \mathbf{u}, \text { curl } \mathbf{v})_{\Omega_{i}}+(\alpha \mathbf{u}, \mathbf{v})_{\Omega_{i}}, \quad \forall \mathbf{u}, \mathbf{v} \in V_{h}\left(\Omega_{i}\right)(i=1,2, \cdots, N) .
$$

The first is the discrete $A_{i}$-extension operator $\mathbf{R}_{h}^{i}: V_{h}\left(\Gamma_{i}\right) \rightarrow V_{h}\left(\Omega_{i}\right)$ defined as follows: For any $\Phi \in V_{h}\left(\Gamma_{i}\right), \mathbf{R}_{h}^{i} \Phi$ satisfies $\mathbf{R}_{h}^{i} \Phi \times \mathbf{n}=\Phi$ on $\Gamma_{i}$ and solves

$$
\left(A_{i} \mathbf{R}_{h}^{i} \Phi, \mathbf{v}_{h}\right)=0, \quad \forall \mathbf{v}_{h} \in V_{h}^{0}\left(\Omega_{i}\right) .
$$

The second is the discrete harmonic extension operator $R_{h}^{i}: Z_{h}\left(\Gamma_{i}\right) \rightarrow Z_{h}\left(\Omega_{i}\right)$ defined as follows: For any $w_{h} \in Z_{h}\left(\Gamma_{i}\right), R_{h}^{i} w_{h} \in Z_{h}\left(\Omega_{i}\right)$ satisfies $R_{h}^{i} w=w_{h}$ on $\Gamma_{i}$ and solves

$$
\left(\nabla R_{h}^{i} w_{h}, \nabla v_{h}\right)=0, \quad \forall v_{h} \in Z_{h}\left(\Omega_{i}\right) \cap H_{0}^{1}\left(\Omega_{i}\right)
$$

\section{NONOVERLAPPING DOMAIN DECOMPOSITION METHODS}

This section addresses how to solve the saddle-point problem (2.2) effectively. For convenience, we introduce two operators $\bar{A}: V_{h}(\Omega) \rightarrow V_{h}(\Omega)$ and $B: Z_{h}(\Omega) \rightarrow$ $V_{h}(\Omega)$ by

$$
\begin{aligned}
& \left(\bar{A} \mathbf{u}_{h}, \mathbf{v}_{h}\right)=\left(\alpha \operatorname{curl} \mathbf{u}_{h}, \text { curl } \mathbf{v}_{h}\right), \quad \forall \mathbf{u}_{h}, \mathbf{v}_{h} \in V_{h}(\Omega), \\
& \left(B p_{h}, \mathbf{v}_{h}\right)=\left(\nabla p_{h}, \beta \mathbf{v}_{h}\right), \quad \forall p_{h} \in Z_{h}(\Omega), \mathbf{v}_{h} \in V_{h}(\Omega) .
\end{aligned}
$$

The dual operator $B^{t}: V_{h}(\Omega) \rightarrow Z_{h}(\Omega)$ of $B$ can be defined by

$$
\left(B^{t} \mathbf{u}_{h}, q_{h}\right)=\left(\beta \mathbf{u}_{h}, \nabla q_{h}\right), \quad \forall q_{h} \in Z_{h}(\Omega) .
$$

Let $\overline{\mathbf{f}}_{h} \in V_{h}(\Omega)$ and $g_{h} \in Z_{h}(\Omega)$ be the $L^{2}$-projections of $\mathbf{f}$ and $g$. Then, the system (2.2) can be written as

$$
\left\{\begin{array}{c}
\left(\bar{A}+\gamma_{0} \beta I\right) \mathbf{u}_{h}+B p_{h}=\overline{\mathbf{f}}_{h}, \\
B^{t} \mathbf{u}_{h}=g_{h} .
\end{array}\right.
$$

In recent years, there has been increasing interest in solving saddle-point problems like (3.1) by iterative methods; see, for example, [5], 6], [17], and [24]. But the most existing methods require the stiffness matrix corresponding to the primal variable $\mathbf{u}_{h}$ above to be nonsingular, so they cannot be applied to solve the saddlepoint system (3.1) with $\gamma_{0}=0$, as the operator $\bar{A}$ is singular in the space $V_{h}(\Omega)$. To overcome this difficulty, we shall introduce another saddle-point system which has the same solution as problem (3.1) when $\gamma_{0}=0$, but which can be solved by existing preconditioned iterative methods.

3.1. Augmented saddle-point system and Uzawa iterative methods. Let $\hat{C}: Z_{h}(\Omega) \rightarrow Z_{h}(\Omega)$ be symmetric and positive definite and chosen as a preconditioner for the discrete Laplace operator on $Z_{h}(\Omega)$. Define

$$
A=\left\{\begin{array}{ll}
\bar{A}+\gamma_{0} \beta I & \text { if } \gamma_{0} \neq 0, \\
\bar{A}+r_{0} B \hat{C}^{-1} B^{t} & \text { if } \gamma_{0}=0,
\end{array} \quad \mathbf{f}_{h}= \begin{cases}\overline{\mathbf{f}}_{h} & \text { if } \gamma_{0} \neq 0, \\
\overline{\mathbf{f}}_{h}+r_{0} B \hat{C}^{-1} g_{h} & \text { if } \gamma_{0}=0,\end{cases}\right.
$$

where $r_{0}$ is some positive constant. One of the possible choices for $r_{0}$ is the average value of $c(x)=\alpha(x) / \beta(x)$. 
Clearly, the system (3.1) has the same solution as the augmented saddle-point problem:

$$
\left\{\begin{array}{l}
A \mathbf{u}_{h}+B p_{h}=\mathbf{f}_{h}, \\
B^{t} \mathbf{u}_{h}=g_{h} .
\end{array}\right.
$$

Let $\hat{A}$ be a preconditioner for $A$. Since the operator $A$ is symmetric and positive definite, the system (3.2) can be solved by many existing iterative methods. Below is a recently developed Uzawa-type algorithm with variable relaxation parameters (see [17 and [18):

Step 1. Choose a parameter $\omega_{i}$ and compute

$$
\mathbf{u}_{h}^{i+1}=\mathbf{u}_{h}^{i}+\omega_{i} \hat{A}^{-1}\left[f_{h}-\left(A \mathbf{u}_{h}^{i}+B p_{h}^{i}\right)\right] .
$$

Step 2. Choose a parameter $\tau_{i}$ and compute

$$
p_{h}^{i+1}=p_{h}^{i}+\tau_{i} \hat{C}^{-1}\left(B^{t} \mathbf{u}_{h}^{i+1}-g_{h}\right) .
$$

Remark 3.1. Some choices of the parameters $\omega_{i}$ and $\tau_{i}$ are given in [17] and [18] to ensure the convergence of the algorithm. Note the fact that

$$
f_{h}-A \mathbf{u}_{h}^{i}=\bar{f}_{h}-\bar{A} \mathbf{u}_{h}^{i}-r_{0} B\left[\hat{C}^{-1}\left(B^{t} \mathbf{u}_{h}^{i}-g_{h}\right)\right]
$$

in the case of $\gamma_{0}=0$, so the value $\hat{C}^{-1}\left(B^{t} \mathbf{u}_{h}^{i}-g_{h}\right)$ computed in Step 2 of the $i$-th iteration can be used in Step 1 of the $(i+1)$-th iteration. That is, the newly added term $r_{0} B \hat{C}^{-1} B^{t}$ in the augmented saddle-point system (3.2) does not create any extra cost in the above Uzawa algorithm as the action of $\hat{C}^{-1}$ is needed only once at each iteration.

The convergence rate of the above Uzawa algorithm is completely determined by the condition numbers $\kappa\left(\hat{A}^{-1} A\right)$ and $\kappa\left(\hat{C}^{-1} B^{t} \hat{A}^{-1} B\right)$; see [17] and [18] for the detailed analyses. In the following we will construct an efficient preconditioner $\hat{A}$ which makes these two condition numbers to be nearly optimal.

3.2. Construction of the preconditioner $\hat{A}$. In the sequel, we shall frequently use the notation $\lesssim$ and $\underset{\sim}{\approx}$. For any two nonnegative quantities $x$ and $y, x \lesssim y$ means that $x \leq C y$ for some constant $C$ independent of mesh size $h$, subdomain size $d$ and the related parameters; $x \underset{\approx}{\sim} y$ means $x \lesssim y$ and $y \lesssim x$.

The proofs of all results in this section will be given in Section 5 .

Let $\tilde{A}: V_{h}(\Omega) \rightarrow V_{h}(\Omega)$ be an operator defined by

$$
(\tilde{A} \mathbf{u}, \mathbf{v})=(\alpha \operatorname{curl} \mathbf{u}, \operatorname{curl} \mathbf{v})+(\alpha \mathbf{u}, \mathbf{v}), \quad \mathbf{u}, \mathbf{v} \in V_{h}(\Omega) .
$$

Theorem 3.1. Let $G(\cdot) \geq 1$ be some given function, and let the operator $\hat{C}$ satisfy

$$
(\beta \nabla \phi, \nabla \phi) \lesssim(\hat{C} \phi, \phi) \lesssim G(d / h)(\beta \nabla \phi, \nabla \phi), \quad \forall \phi \in Z_{h}(\Omega) .
$$

Then we have

$$
\frac{1}{G(d / h)}\left(\tilde{A} \mathbf{v}_{h}, \mathbf{v}_{h}\right) \lesssim\left(A \mathbf{v}_{h}, \mathbf{v}_{h}\right) \lesssim\left(\tilde{A} \mathbf{v}_{h}, \mathbf{v}_{h}\right), \quad \forall \mathbf{v}_{h} \in V_{h}(\Omega) .
$$

With this theorem, it suffices to construct a preconditioner for $\tilde{A}$ instead of $A$. 
We first define two subspaces of $V_{h}(\Omega)$ :

$$
V^{p}(\Omega)=\left\{\mathbf{v} \in V_{h}(\Omega) ; \mathbf{v} \times \mathbf{n}=\mathbf{0} \text { on } \Gamma\right\}=\prod_{k=1}^{N} V_{h}^{0}\left(\Omega_{k}\right),
$$

$V^{H}(\Omega)=\left\{\mathbf{v} \in V_{h}(\Omega) ; \mathbf{v}\right.$ is the discrete $A_{i}$-extension of $\left.\mathbf{v}\right|_{\partial \Omega_{i}}$ in each $\left.\Omega_{i}\right\}$.

Obviously, $V_{h}(\Omega)$ has the orthogonal decomposition with respect to the inner product $(\tilde{A} \cdot, \cdot)$ :

$$
V_{h}(\Omega)=V^{p}(\Omega) \oplus V^{H}(\Omega) .
$$

Furthermore, we define two subspaces of $V^{H}(\Omega)$ :

$$
\begin{gathered}
V^{i j}(\Omega)=\left\{\mathbf{v} \in V^{H}(\Omega) ; \operatorname{supp}(\mathbf{v}) \subset \Omega_{i j}=\Omega_{i} \cup \Omega_{j} \cup \Gamma_{i j}\right\}, \\
V^{0}(\Omega)=\left\{\mathbf{v} \in V^{H}(\Omega) ; \lambda_{e}(\mathbf{v})=0 \text { for each } e \in \mathrm{F}_{\partial} \text { with } \mathrm{F} \subset \Gamma\right\} .
\end{gathered}
$$

The subspace $V^{0}(\Omega)$ is called the coarse subspace. The introduction of such a coarse subspace is based on the following consideration: for any $\mathbf{v}_{h} \in V_{h}(\Omega)$, its tangential components are continuous on all cross-edges, namely, the edges which are shared by more than two fine elements (in the two-dimensional case, the tangential components have no definitions at the cross-points), but the moments on the cross-edges are not sufficient to determine the values of the tangential trace $\mathbf{v}_{h} \times \mathbf{n}$ on these edges.

It is easy to see that the space $V_{h}(\Omega)$ has the (nondirect sum) decomposition

$$
V_{h}(\Omega)=V^{p}(\Omega) \oplus\left(V^{0}(\Omega)+\sum_{\Gamma_{i j}} V^{i j}(\Omega)\right) .
$$

Next, we define the corresponding solvers on the subspaces $V^{p}(\Omega), V^{0}(\Omega)$ and $V^{i j}(\Omega)$.

Let $\hat{A}_{p}: V^{p}(\Omega) \rightarrow V^{p}(\Omega)$ and $\hat{A}_{i j}: V^{i j}(\Omega) \rightarrow V^{i j}(\Omega)$ be symmetric and positive definite operators such that

$$
\left(\hat{A}_{p} \mathbf{v}, \mathbf{v}\right) \equiv \sum_{k=1}^{N}\left(A_{k} \mathbf{v}_{k}, \mathbf{v}_{k}\right)_{\Omega_{k}}, \quad \forall \mathbf{v} \in V^{p}(\Omega),
$$

where $\mathbf{v}_{k}=\left.\mathbf{v}\right|_{\Omega_{k}}$ for $k=1,2, \cdots, N$ and

$$
\left(\hat{A}_{i j} \mathbf{v}, \mathbf{v}\right) \underset{\sim}{\sim}\left(A_{i} \mathbf{v}_{i}, \mathbf{v}_{i}\right)_{\Omega_{i}}+\left(A_{j} \mathbf{v}_{j}, \mathbf{v}_{j}\right)_{\Omega_{j}}, \quad \forall \mathbf{v} \in V^{i j}(\Omega) .
$$

The global coarse solvers should be solvable in an efficient way on $V^{0}(\Omega)$, and their constructions are much more tricky and technical than the local solvers. To do so, we introduce the so-called tangential divergence $\operatorname{div}_{\tau} \Phi$ of any $\Phi \in V_{h}\left(\Gamma_{i}\right)$, as done in 1 and 2 .

For ease of notation, we assume that $\alpha(x)=\alpha_{i}$ for $x \in \Omega_{i}$, with $\alpha_{i}$ being constants.

Then we define the coarse solver $\hat{A}_{0}: V^{0}(\Omega) \rightarrow V^{0}(\Omega)$ as follows:

$$
\begin{aligned}
\left(\hat{A}_{0} \mathbf{v}, \mathbf{w}\right)= & h[1+\log (d / h)] \\
& \times \sum_{i=1}^{N} \alpha_{i}\left\{\left\langle\left.\operatorname{div}_{\tau}(\mathbf{v} \times \mathbf{n})\right|_{\Gamma_{i}},\left.\operatorname{div}_{\tau}(\mathbf{w} \times \mathbf{n})\right|_{\Gamma_{i}}\right\rangle_{\Delta_{i}}+\langle\mathbf{v} \times \mathbf{n}, \mathbf{w} \times \mathbf{n}\rangle_{\Delta_{i}}\right\} .
\end{aligned}
$$


Let $Q_{p}: V^{p}(\Omega) \rightarrow V^{p}(\Omega), Q_{0}: V_{h}(\Omega) \rightarrow V^{0}(\Omega)$ and $Q_{i j}: V_{h}(\Omega) \rightarrow V^{i j}(\Omega)$ denote the $L^{2}$-projections. The preconditioner for $\hat{A}$ can be defined as follows:

$$
\hat{A}^{-1}=\hat{A}_{p}^{-1} Q_{p}+\hat{A}_{0}^{-1} Q_{0}+\sum_{\Gamma_{i j}} \hat{A}_{i j}^{-1} Q_{i j}
$$

For this preconditioner, we have

Theorem 3.2. The condition number of the preconditioned system can be estimated by

$$
\operatorname{cond}\left(\hat{A}^{-1} A\right) \lesssim G(d / h)[1+\log (d / h)]^{2} .
$$

Remark 3.2. It is known that $\left.\operatorname{div}_{\tau}\left(L_{e} \times \mathbf{n}\right)\right|_{\Gamma_{i}}$ vanishes for any interior edge $e$ of $\Omega_{i}$. Moreover, we have $\left.\operatorname{div}_{\tau}\left(L_{e} \times \mathbf{n}\right)\right|_{\Gamma_{i}}=\left.\left(\operatorname{curl} L_{e}\right) \cdot \mathbf{n}\right|_{\Gamma_{i}}$ for each $e \subset \Gamma_{i}$. Thus, the entries of the stiffness matrix of $\hat{A}_{0}$ are of the form:

$$
\begin{array}{r}
h[1+\log (d / h)] \sum_{i=1}^{N} \alpha_{i}\left\{\left\langle\left(\operatorname{curl} L_{e}\right) \cdot \mathbf{n},\left(\operatorname{curl} L_{e^{\prime}}\right) \cdot \mathbf{n}\right\rangle_{\Delta_{i}}+\left\langle L_{e} \times \mathbf{n}, L_{e^{\prime}} \times \mathbf{n}\right\rangle_{\Delta_{i}}\right\}, \\
e, e^{\prime} \in \bigcup_{\mathrm{F} \subset \Gamma} \mathrm{F}_{b} .
\end{array}
$$

The coarse solver $\hat{A}_{0}$ involves computations only on $\Delta_{i}$, a very small fraction of the interface $\Gamma$. Also, $\hat{A}_{0}$ is rather simple in comparison with coarse solvers in many existing substructuring preconditioners for standard elliptic problems, where some optimizations are involved [4], 32]. The preconditioner $\hat{A}$ can be implemented as in [4] and 32].

For the preconditioned Schur complement, we have

Theorem 3.3. The condition number of the preconditioned Schur complement system can be estimated by

$$
\operatorname{cond}\left(\hat{C}^{-1} B^{t} \hat{A}^{-1} B\right) \lesssim G(d / h)[1+\log (d / h)]^{2} .
$$

Remark 3.3. When $\hat{C}$ is chosen as the usual multigrid preconditioner, we have $G(d / h)=1$; when $\hat{C}$ is chosen as the substructuring preconditioner (see [4], [32]), we have $G(d / h)=[1+\log (d / h)]^{2}$.

\section{Some AUXiLiary Lemmata}

As we shall see, the proof of Theorem 3.2 namely, the estimate of the condition number for the preconditioned system, is very technical. This section presents some basic properties of Sobolev spaces and some auxiliary lemmata, which will be used to deal with the technical difficulties in the proof of Theorem 3.2 .

4.1. Helmholz decomposition and edge element interpolation. Denote by $H\left(\operatorname{curl} ; \Omega_{i}\right)$ the restriction of $H_{0}(\operatorname{curl} ; \Omega)$ on the subdomain $\Omega_{i}$. It is known that the spaces $H_{0}(\mathbf{c u r l} ; \Omega)$ and $H\left(\operatorname{curl} ; \Omega_{i}\right)$ can be decomposed into (see 12])

$$
\begin{aligned}
H_{0}(\operatorname{curl} ; \Omega) & =\nabla H_{0}^{1}(\Omega) \oplus H_{0}^{\perp}(\operatorname{curl} ; \Omega), \\
H\left(\operatorname{curl} ; \Omega_{i}\right) & =\nabla H^{1}\left(\Omega_{i}\right) \oplus H^{\perp}\left(\operatorname{curl} ; \Omega_{i}\right),
\end{aligned}
$$

where

$$
H_{0}^{\perp}(\operatorname{curl} ; \Omega)=\left\{\mathbf{v} \in H_{0}(\operatorname{curl} ; \Omega): \operatorname{div} \mathbf{v}=0\right\},
$$




$$
H^{\perp}\left(\mathbf{c u r l} ; \Omega_{i}\right)=\left\{\mathbf{v} \in H\left(\operatorname{curl} ; \Omega_{i}\right): \operatorname{div} \mathbf{v}=0, \mathbf{v} \cdot \mathbf{n}=0 \text { on } \Gamma_{i}\right\} .
$$

It follows from (Theorem 4.3, [2] ) that $H_{0}^{\perp}(\operatorname{curl} ; \Omega) \subset H^{\delta}(\Omega)^{3}$ for some $1 / 2<\delta<1$, and

$$
\|\mathbf{v}\|_{\delta, \Omega} \lesssim\|\operatorname{curl} \mathbf{v}\|_{0, \Omega}, \quad \forall \mathbf{v} \in H_{0}^{\perp}(\operatorname{curl} ; \Omega) .
$$

Since $\Omega_{i}$ is a convex polyhedron, $H^{\perp}\left(\operatorname{curl} ; \Omega_{i}\right) \subset H^{1}\left(\Omega_{i}\right)^{3}$ (see [12]). Moreover, we have

$$
d^{-2}\|\mathbf{v}\|_{0, \Omega_{i}}+|\mathbf{v}|_{1, \Omega_{i}} \lesssim\|\mathbf{c u r l} \mathbf{v}\|_{0, \Omega_{i}}, \quad \forall \mathbf{v} \in H^{\perp}\left(\operatorname{curl} ; \Omega_{i}\right) .
$$

Here and in what follows, for any given domain $D$ and each integer $m \geq 0$ we use $H^{m}(D)$ to denote the standard Sobolev space of real functions with their weak derivatives of order up to $m$ in the Lebesgue space $L^{2}(D)$, and we use $\|\cdot\|_{m, D}$ and $|\cdot|_{m, D}$ to denote its norm and semi-norm. For a fractional number $s$, the Sobolev space $H^{s}(\Omega)$ is defined by the standard interpolation theory.

For a $\mathbf{v}_{h} \in V_{h}(\Omega)$, using (4.1) and (4.3) we can decompose $\mathbf{v}_{h}$ as follows:

$$
\mathbf{v}_{h}=\nabla p \oplus \mathbf{w} \quad \text { in } \Omega
$$

where $\mathbf{w} \in H_{0}^{\perp}(\operatorname{curl} ; \Omega) \cap H^{\delta}(\Omega)^{3}$ for some $\delta>\frac{1}{2}$ and $p \in H_{0}^{1}(\Omega)$ solves

$$
(\nabla p, \nabla q)=\left(\mathbf{v}_{h}, \nabla q\right), \quad \forall q \in H_{0}^{1}(\Omega) .
$$

Similarly, for a $\mathbf{v}_{h} \in V_{h}\left(\Omega_{i}\right)$, it follows from (4.2) and (4.4) that

$$
\mathbf{v}_{h}=\nabla p \oplus \mathbf{w} \quad \text { in } \Omega_{i}
$$

where $\mathbf{w} \in H^{\perp}\left(\operatorname{curl} ; \Omega_{i}\right) \cap H^{1}\left(\Omega_{i}\right)^{3}$, and $p \in H^{1}\left(\Omega_{i}\right)$ solves

$$
(\nabla p, \nabla q)_{\Omega_{i}}=\left(\mathbf{v}_{h}, \nabla q\right)_{\Omega_{i}}, \quad \forall q \in H^{1}\left(\Omega_{i}\right) .
$$

Next, we present some interpolation results related to the finite element space $V_{h}(\Omega)$. We know from [3] (Lemma 4.7) that for any $\mathbf{v} \in H^{\delta}(\Omega)$ with curl v $\in$ $L^{p}(\Omega)^{3}\left(\delta>\frac{1}{2}\right.$ and $\left.p>2\right)$ we can define its interpolant $\mathbf{r}_{h} \mathbf{v}$ in $V_{h}(\Omega)$ by the relation

$$
\lambda_{e}\left(\mathbf{r}_{h} \mathbf{v}\right)=\int_{e} \mathbf{v} \cdot \mathbf{t}_{e} d s, \quad \forall e \in \mathcal{E}_{h} .
$$

Lemma 4.1 can be found in [9]:

Lemma 4.1. Assume that $\mathbf{w}$ and curl $\mathbf{w}$ are both in $H^{\delta}\left(\Omega_{i}\right)$ for some $\delta>\frac{1}{2}$. Then,

$$
\left\|\mathbf{r}_{h} \mathbf{w}-\mathbf{w}\right\|_{0, \Omega_{i}}+\left\|\operatorname{curl}\left(\mathbf{r}_{h} \mathbf{w}-\mathbf{w}\right)\right\|_{0, \Omega_{i}} \lesssim h^{\delta}\left(\|\mathbf{w}\|_{\delta, \Omega_{i}}+\|\operatorname{curl} \mathbf{w}\|_{\delta, \Omega_{i}}\right) .
$$

Lemma 4.2. For any $\mathbf{v}_{h} \in V_{h}(\Omega)$ which satisfies

$$
\left(\beta \mathbf{v}_{h}, \nabla q_{h}\right)=0, \quad \forall q_{h} \in Z_{h}(\Omega),
$$

we have

$$
\left\|\beta^{\frac{1}{2}} \mathbf{v}_{h}\right\|_{0, \Omega} \lesssim \| \beta^{\frac{1}{2}} \text { curl } \mathbf{v}_{h} \|_{0, \Omega}
$$

Proof. Let $\mathbf{w} \in H_{0}^{\perp}(\operatorname{curl} ; \Omega)$ be defined by (4.5). Then, we have (see [12])

$$
\mathbf{v}_{h}=\mathbf{r}_{h} \nabla p+\mathbf{r}_{h} \mathbf{w}=\nabla p_{h}+\mathbf{r}_{h} \mathbf{w},
$$

with $p_{h} \in Z_{h}(\Omega)$. Since $\left(\beta \mathbf{v}_{h}, \nabla p_{h}\right)=0$, we infer from (4.10) that

$$
\left\|\beta^{\frac{1}{2}} \nabla p_{h}\right\|_{0, \Omega} \leq\left\|\beta^{\frac{1}{2}} \mathbf{r}_{h} \mathbf{w}\right\|_{0, \Omega} .
$$


This, together with (4.10), leads to

$$
\begin{aligned}
\left\|\beta^{\frac{1}{2}} \mathbf{v}_{h}\right\|_{0, \Omega} & \leq\left\|\beta^{\frac{1}{2}} \nabla p_{h}\right\|_{0, \Omega}+\left\|\beta^{\frac{1}{2}} \mathbf{r}_{h} \mathbf{w}\right\|_{0, \Omega} \\
& \lesssim\left\|\beta^{\frac{1}{2}} \mathbf{r}_{h} \mathbf{w}\right\|_{0, \Omega} \leq\left\|\beta^{\frac{1}{2}} \mathbf{w}\right\|_{0, \Omega}+\left\|\beta^{\frac{1}{2}}\left(\mathbf{r}_{h} \mathbf{w}-\mathbf{w}\right)\right\|_{0, \Omega}
\end{aligned}
$$

It follows from (4.3) that

$$
\left\|\beta^{\frac{1}{2}} \mathbf{w}\right\|_{0, \Omega} \lesssim\left\|\beta^{\frac{1}{2}} \operatorname{curl} \mathbf{w}\right\|_{0, \Omega}=\left\|\beta^{\frac{1}{2}} \operatorname{curl} \mathbf{v}_{h}\right\|_{0, \Omega} .
$$

On the other hand, following the proof of Lemma 3.2 in [9], we derive

$$
\begin{aligned}
\left\|\beta^{\frac{1}{2}}\left(\mathbf{r}_{h} \mathbf{w}-\mathbf{w}\right)\right\|_{0, K} & \lesssim h^{\delta}\left(\left\|\beta^{\frac{1}{2}} \mathbf{w}\right\|_{H^{\delta}(K)}+\left\|\beta^{\frac{1}{2}} \operatorname{curl} \mathbf{w}\right\|_{H^{\delta}(K)}\right) \\
& \lesssim\left\|\beta^{\frac{1}{2}} \operatorname{curlv}_{h}\right\|_{0, K}, \quad \forall K \in \mathcal{T}_{h},
\end{aligned}
$$

where we have also used (4.3) and the inverse inequality. This with (4.11) gives the desired result.

Lemma 4.3. For any $\mathbf{v}_{h} \in V_{h}\left(\Omega_{i}\right)$, let $\mathbf{w}$ be defined as in (4.6). Then

$$
\left\|\mathbf{r}_{h} \mathbf{w}-\mathbf{w}\right\|_{0, \Omega_{i}} \lesssim h\left\|\operatorname{curl}_{\mathbf{v}_{h}}\right\|_{0, \Omega_{i}} .
$$

Proof. For any element $K \in \mathcal{T}_{h}$, let $\mathbf{x}=F_{K} \hat{\mathbf{x}}=B_{K} \hat{\mathbf{x}}+b_{K}$ be the affine mapping between $K$ and the reference element $\hat{K}$. In $\hat{K}$, define

$$
\hat{\mathbf{w}}=B_{K}^{t} \mathbf{w} \circ F_{K}, \quad \hat{\mathbf{v}}_{h}=B_{K}^{t} \mathbf{v}_{h} \circ F_{K} .
$$

Let $\hat{\mathbf{r}}_{h}$ be the interpolant on the reference element $\hat{K}$. One can show that (cf. 9])

$$
\left\|\hat{\mathbf{r}}_{h} \hat{\mathbf{w}}-\hat{\mathbf{w}}\right\|_{0, \hat{K}} \lesssim\left(\|\hat{\mathbf{w}}\|_{\delta, \hat{K}}^{2}+\|\widehat{\operatorname{curl}} \hat{\mathbf{w}}\|_{\delta, \hat{K}}^{2}\right)^{\frac{1}{2}} .
$$

Since $\widehat{\operatorname{curl}} \hat{\mathbf{w}}=\widehat{\operatorname{curl}} \hat{\mathbf{v}}_{h}$, we have

$$
\left\|\hat{\mathbf{r}}_{h} \hat{\mathbf{w}}-\hat{\mathbf{w}}\right\|_{0, \hat{K}} \lesssim\left(\|\hat{\mathbf{w}}\|_{1, \hat{K}}^{2}+\left\|\widehat{\operatorname{curl}} \hat{\mathbf{v}}_{h}\right\|_{0, \hat{K}}^{2}\right)^{\frac{1}{2}} .
$$

As the interpolation operator $\hat{\mathbf{r}}_{h}$ preserves constants, it follows by 4.15) that

$$
\left\|\hat{\mathbf{r}}_{h} \hat{\mathbf{w}}-\hat{\mathbf{w}}\right\|_{0, \hat{K}} \lesssim\left(|\hat{\mathbf{w}}|_{1, \hat{K}}^{2}+\left\|\widehat{\operatorname{curl}} \mathbf{l} \hat{\mathbf{v}}_{h}\right\|_{0, \hat{K}}^{2}\right)^{)^{\frac{1}{2}}} .
$$

Now, by the standard scaling technique we obtain

$$
\begin{aligned}
\left\|\mathbf{r}_{h} \mathbf{w}-\mathbf{w}\right\|_{0, K}^{2} & \lesssim h\left\|\hat{\mathbf{r}}_{h} \hat{\mathbf{w}}-\hat{\mathbf{w}}\right\|_{0, \hat{K}}^{2} \\
& \lesssim h\left(|\hat{\mathbf{w}}|_{1, \hat{K}}^{2}+\left\|\mathbf{c u r l} \hat{\mathbf{v}}_{h}\right\|_{0, \hat{K}}^{2}\right) \\
& \lesssim h^{2}\left(|\mathbf{w}|_{1, K}^{2}+\left\|\mathbf{c u r l} \mathbf{v}_{h}\right\|_{0, K}^{2}\right),
\end{aligned}
$$

which, together with (4.4), immediately gives the desired result.

Lemma 4.4. For any $\mathbf{v}_{h} \in V_{h}\left(\Omega_{i}\right)$, let $\mathbf{w}$ be defined by (4.6) and let $p_{h} \in Z_{h}\left(\Omega_{i}\right)$ be defined by

$$
\mathbf{v}_{h}=\mathbf{r}_{h}(\nabla p \oplus \mathbf{w})=\nabla p_{h}+\mathbf{r}_{h} \mathbf{w} \quad \text { in } \Omega_{i} .
$$

Then we have

$$
\left|p_{h}\right|_{1, \Omega_{i}} \lesssim\left\|\mathbf{v}_{h}\right\|_{0, \Omega_{i}}+\max \left\{h, d^{2}\right\}\left\|\boldsymbol{c u r l} \mathbf{v}_{h}\right\|_{0, \Omega_{i}}
$$


Proof. We know from (4.15) that for any $K \in \mathcal{T}_{h}$,

$$
\left\|\hat{\mathbf{r}}_{h} \hat{\mathbf{w}}\right\|_{0, \hat{K}}^{2} \lesssim\|\hat{\mathbf{w}}\|_{1, \hat{K}}^{2}+\left\|\widehat{\operatorname{curl}} \hat{\mathbf{v}}_{h}\right\|_{0, \hat{K}}^{2} .
$$

Therefore, we have

$$
\left\|\mathbf{r}_{h} \mathbf{w}\right\|_{0, K}^{2} \lesssim h\left\|\hat{\mathbf{r}}_{h} \hat{\mathbf{w}}\right\|_{0, \hat{K}}^{2} \lesssim h^{2}\left\|\mathbf{c u r l} \mathbf{v}_{h}\right\|_{0, K}^{2}+h^{2}|\mathbf{w}|_{1, K}^{2}+\|\mathbf{w}\|_{0, K}^{2},
$$

which with (4.4) leads to

$$
\begin{aligned}
\left\|\mathbf{r}_{h} \mathbf{w}\right\|_{0, \Omega_{i}}^{2} & \lesssim h^{2}\left\|\mathbf{c u r l} \mathbf{v}_{h}\right\|_{0, \Omega_{i}}^{2}+h^{2}|\mathbf{w}|_{1, \Omega_{i}}^{2}+\|\mathbf{w}\|_{0, \Omega_{i}}^{2} \\
& \lesssim \max \left\{h^{2}, d^{4}\right\}\left\|\mathbf{c u r l} \mathbf{v}_{h}\right\|_{0, \Omega_{i}}^{2} .
\end{aligned}
$$

The desired estimate now follows from this and the triangle inequality

$$
\left\|\nabla p_{h}\right\|_{0, \Omega_{i}} \leq\left\|\mathbf{v}_{h}\right\|_{0, \Omega_{i}}+\left\|\mathbf{r}_{h} \mathbf{w}\right\|_{0, \Omega_{i}} .
$$

4.2. Some scaled norms and their estimates. A large part of the condition number estimates in Section 5 will be carried out on the subdomains, for which we need some scaled norms. For the space $H^{1}\left(\Omega_{i}\right)^{3}$, we define a scaled norm by

$$
\|\mathbf{v}\|_{1, \Omega_{i}}=\left\{|\mathbf{v}|_{1, \Omega_{i}}^{2}+d^{-2}\|\mathbf{v}\|_{0, \Omega_{i}}^{2}\right\}^{\frac{1}{2}}, \quad \forall \mathbf{v} \in H^{1}\left(\Omega_{i}\right)^{3},
$$

while for the space $H\left(\mathbf{c u r l} ; \Omega_{i}\right)$, we define its scaled norm by

$$
\|\mathbf{u}\|_{\text {curl } ; \Omega_{i}}=\left\{\|\operatorname{curl} \mathbf{u}\|_{0, \Omega_{i}}^{2}+d^{-2}\|\mathbf{u}\|_{0, \Omega_{i}}^{2}\right\}^{\frac{1}{2}}
$$

and for each $\lambda \in H^{-\frac{1}{2}}\left(\Gamma_{i}\right)$, we define

$$
\|\lambda\|_{-\frac{1}{2}, \Gamma_{i}}=\sup _{v \in H^{\frac{1}{2}}\left(\Gamma_{i}\right)} \frac{\langle\lambda, v\rangle_{\Gamma_{i}}}{\|v\|_{\frac{1}{2}, \Gamma_{i}}}
$$

where

$$
\|v\|_{\frac{1}{2}, \Gamma_{i}}=\left(|v|_{\frac{1}{2}, \Gamma_{i}}^{2}+d^{-1}\|v\|_{0, \Gamma_{i}}^{2}\right)^{\frac{1}{2}} .
$$

The same notation will be used for the norms in the space $H^{-\frac{1}{2}}\left(\Gamma_{i}\right)^{3}$.

For any $\Phi \in V_{h}\left(\Gamma_{i}\right)$, recall that $\operatorname{div}_{\tau} \Phi$ is the tangential divergence of $\Phi$. We know that $\operatorname{div}_{\tau} \Phi \in H^{-\frac{1}{2}}\left(\Gamma_{i}\right)$ (cf. [1], [2]). This leads to the following important norm:

$$
\|\Phi\|_{\mathcal{X}_{\Gamma_{i}}}=d^{-1}\|\Phi\|_{-\frac{1}{2}, \Gamma_{i}}+\left\|\operatorname{div}_{\tau} \Phi\right\|_{-\frac{1}{2}, \Gamma_{i}} .
$$

The following two results about the norm $\|\cdot\|_{\mathcal{X}_{\Gamma_{i}}}$ can be found in [1], [2] (using the standard dilation from the reference domain):

Lemma 4.5. Let $\mathbf{u} \in V_{h}\left(\Omega_{i}\right)$, which satisfies $\mathbf{u} \times \mathbf{n}=\Phi$ on $\Gamma_{i}$. Then

$$
\|\Phi\|_{\mathcal{X}_{\Gamma_{i}}} \lesssim\|\mathbf{u}\|_{\text {curl } ; \Omega_{i}} .
$$

Lemma 4.6. The discrete $A_{i}$-extension $\mathbf{R}_{h}^{i} \Phi \in V_{h}\left(\Omega_{i}\right)$ satisfies

$$
\left\|\mathbf{R}_{h}^{i} \Phi\right\|_{0, \Omega_{i}}+\left\|\operatorname{curl}\left(\mathbf{R}_{h}^{i} \Phi\right)\right\|_{0, \Omega_{i}} \lesssim\|\Phi\|_{\mathcal{X}_{\Gamma_{i}}} .
$$

The following results can be found in [10], while the factors $d^{\alpha}$ are derived by the standard scaling argument: 
Lemma 4.7. For any $\mathbf{v} \in H\left(\operatorname{curl} ; \Omega_{i}\right) \cap H\left(\operatorname{div} ; \Omega_{i}\right)$, if $\mathbf{v} \times \mathbf{n}$ or $\mathbf{v} \cdot \mathbf{n}$ is in $L^{2}\left(\Gamma_{i}\right)$, then

$$
\begin{aligned}
& \quad|\mathbf{v}|_{\frac{1}{2}, \Omega_{i}} \lesssim d^{-\frac{1}{2}}\|\mathbf{v}\|_{0, \Omega_{i}}+d^{\frac{1}{2}}\|\operatorname{div} \mathbf{v}\|_{0, \Omega_{i}}+d^{\frac{1}{2}} \| \text { curl } \mathbf{v}\left\|_{0, \Omega_{i}}+\right\| \mathbf{v} \cdot \mathbf{n} \|_{0, \Gamma_{i}} \\
& \|\mathbf{v} \cdot \mathbf{n}\|_{0, \Gamma_{i}} \lesssim d^{-\frac{1}{2}}\|\mathbf{v}\|_{0, \Omega_{i}}+d^{\frac{1}{2}}\|\operatorname{div} \mathbf{v}\|_{0, \Omega_{i}}+d^{\frac{1}{2}} \| \text { curl } \mathbf{v}\left\|_{0, \Omega_{i}}+\right\| \mathbf{v} \times \mathbf{n} \|_{0, \Gamma_{i}} .
\end{aligned}
$$

For any $\Phi \in V_{h}\left(\Gamma_{i}\right)$, let $\omega(\Phi) \in H^{1}\left(\Omega_{i}\right)$ be the weak solution of the problem

$$
\begin{cases}\triangle \omega(\Phi)=0 & \text { in } \Omega_{i} \\ \frac{\partial \omega(\Phi)}{\partial \mathbf{n}}=-\operatorname{div}_{\tau} \Phi & \text { on } \Gamma_{i} \\ \int_{\Omega_{i}} \omega(\Phi) d x=0 . & \end{cases}
$$

Consider the equations

$$
\begin{cases}\operatorname{curl} \operatorname{curl} \mathbf{q}(\Phi)-\nabla \operatorname{div} \mathbf{q}(\Phi)=\nabla \omega(\Phi) & \text { in } \Omega_{i}, \\ \mathbf{n} \times \operatorname{curl} \mathbf{q}(\Phi)=\Phi & \text { on } \Gamma_{i}, \\ \mathbf{q}(\Phi) \cdot \mathbf{n}=0 & \text { on } \Gamma_{i}\end{cases}
$$

Lemma 4.8. Let $\mathbf{q}(\Phi) \in H\left(\mathbf{c u r l} ; \Omega_{i}\right)$ be the solution of the equation (4.23), and let $\tilde{\mathbf{w}}(\Phi)=\operatorname{curl} \mathbf{q}(\Phi)$. Then we have $(1) \mathbf{n} \times \tilde{\mathbf{w}}(\Phi)=\Phi$ on $\Gamma_{i} ;(2) \tilde{\mathbf{w}}(\Phi)$, $\operatorname{curl} \tilde{\mathbf{w}} \in$ $H^{\frac{1}{2}+\delta}\left(\Omega_{i}\right)$ for some $\delta \in\left[0, \frac{1}{2}\right) ;(3) \operatorname{curl} \tilde{\mathbf{w}}(\Phi) \cdot \mathbf{n}=-\operatorname{div}_{\tau} \Phi$ on $\Gamma_{i} ;(4)$ curl curl $\tilde{\mathbf{w}}(\Phi)$ $=0 ;(5) \operatorname{div} \tilde{\mathbf{w}}(\Phi)=0$; and

$$
\begin{aligned}
\|\tilde{\mathbf{w}}(\Phi)\|_{0, \Omega_{i}} & \lesssim\|\Phi\|_{-\frac{1}{2}, \Gamma_{i}}+d\left\|\operatorname{div}_{\tau} \Phi\right\|_{-\frac{1}{2}, \Gamma_{i}}, \\
\| \text { curl } \tilde{\mathbf{w}}(\Phi) \|_{0, \Omega_{i}} & \lesssim\left\|\operatorname{div}_{\tau} \Phi\right\|_{-\frac{1}{2}, \Gamma_{i}} .
\end{aligned}
$$

Proof. The inferences (1) and (5) are obvious. It is shown in [1] that $\operatorname{div} \mathbf{q}(\Phi)=0$ and

$$
\|\nabla \omega(\Phi)\|_{0, \Omega_{i}} \lesssim\left\|\operatorname{div}_{\tau} \Phi\right\|_{-\frac{1}{2}, \Gamma_{i}}
$$

and so by (4.22) and (4.23) we obtain (3), (4) and (4.25). Since $\left.\Phi\right|_{\Gamma_{i}} \in H^{\delta}\left(\Gamma_{i}\right)^{3}$ and $\operatorname{div}_{\tau}\left(\left.\Phi\right|_{\Gamma_{i}}\right) \in H^{\delta}\left(\Gamma_{i}\right)$ for any $\delta \in\left[0, \frac{1}{2}\right)$, the inference (2) follows by (1), (3) and Theorem 4.4 in [2]. Finally for (4.24), using (4.23) and Green's formulas, we have (note that $\operatorname{div} \mathbf{q}(\Phi)=0$ )

$$
\|\operatorname{curl} \mathbf{q}(\Phi)\|_{0, \Omega_{i}}^{2}=(\nabla \omega(\Phi), \mathbf{q}(\Phi))_{\Omega_{i}}-\langle\Phi, \mathbf{q}(\Phi)\rangle_{\Gamma_{i}} .
$$

Now (4.24) follows from the Cauchy-Schwarz inequality and (4.4).

Lemma 4.9 plays a key role in the construction of our coarse solver.

Lemma 4.9. For any $\Phi \in V_{h}\left(\Gamma_{i}\right)$ satisfying

$$
\|\Phi\|_{-\frac{1}{2}, \Gamma_{i}}+\left\|\operatorname{div}_{\tau} \Phi\right\|_{-\frac{1}{2}, \Gamma_{i}} \lesssim h^{\frac{1}{2}}[1+\log (d / h)]^{\frac{1}{2}}\left(\|\Phi\|_{0, \Gamma_{i}}+\left\|\operatorname{div}_{\tau} \Phi\right\|_{0, \Gamma_{i}}\right),
$$

we have

$$
\left\|\mathbf{R}_{h}^{i} \Phi\right\|_{0, \Omega_{i}}+\left\|\operatorname{curl} \mathbf{R}_{h}^{i} \Phi\right\|_{0, \Omega_{i}} \lesssim h^{\frac{1}{2}}[1+\log (d / h)]^{\frac{1}{2}}\left(\|\Phi\|_{0, \Gamma_{i}}+\left\|\operatorname{div}_{\tau} \Phi\right\|_{0, \Gamma_{i}}\right) .
$$

Proof. Let $\tilde{\mathbf{w}}(\Phi) \in H^{\delta}\left(\Omega_{i}\right)$, also with $\operatorname{curl} \tilde{\mathbf{w}} \in H^{\delta}\left(\Omega_{i}\right)$ for some $\delta>\frac{1}{2}$, be defined as in Lemma 4.8. It follows from Lemma 4.8(1) that $\mathbf{r}_{h} \tilde{\mathbf{w}}(\Phi) \times \mathbf{n}=\Phi$ on $\Gamma_{i}$. By the minimum energy property of the discrete $A_{i}$-extension, we have

$$
\left\|\mathbf{R}_{h}^{i} \Phi\right\|_{0, \Omega_{i}}+\left\|\operatorname{curl}\left(\mathbf{R}_{h}^{i} \Phi\right)\right\|_{0, \Omega_{i}} \leq\left\|\mathbf{r}_{h} \tilde{\mathbf{w}}(\Phi)\right\|_{0, \Omega_{i}}+\left\|\operatorname{curl}\left(\mathbf{r}_{h} \tilde{\mathbf{w}}(\Phi)\right)\right\|_{0, \Omega_{i}} .
$$


It suffices to prove

$\left\|\mathbf{r}_{h} \tilde{\mathbf{w}}(\Phi)\right\|_{0, \Omega_{i}}+\left\|\operatorname{curl}\left(\mathbf{r}_{h} \tilde{\mathbf{w}}(\Phi)\right)\right\|_{0, \Omega_{i}} \lesssim h^{\frac{1}{2}}[1+\log (d / h)]^{\frac{1}{2}}\left(\|\Phi\|_{0, \Gamma_{i}}+\left\|\operatorname{div}_{\tau} \Phi\right\|_{0, \Gamma_{i}}\right)$.

By (4.2), $\tilde{\mathbf{w}}(\Phi)$ can be decomposed as follows:

$$
\tilde{\mathbf{w}}(\Phi)=\nabla p(\Phi) \oplus \mathbf{w}(\Phi), \quad \mathbf{w}(\Phi) \in H^{\perp}\left(\mathbf{c u r l} ; \Omega_{i}\right)
$$

with $p(\Phi)$ satisfying the equations (cf. Lemma $4.8(5)$ )

$$
\begin{cases}\triangle p(\Phi)=\operatorname{div} \tilde{\mathbf{w}}(\Phi)=0 & \text { in } \Omega_{i} \\ \frac{\partial p(\Phi)}{\partial \mathbf{n}}=\tilde{\mathbf{w}}(\Phi) \cdot \mathbf{n} & \text { on } \Gamma_{i}, \\ \int_{\Omega_{i}} p(\Phi) d x=0 . & \end{cases}
$$

We have by Lemma 4.1 that

$$
\left\|\mathbf{r}_{h} \mathbf{w}(\Phi)-\mathbf{w}(\Phi)\right\|_{0, \Omega_{i}}+\left\|\operatorname{curl}\left(\mathbf{r}_{h} \mathbf{w}(\Phi)-\mathbf{w}(\Phi)\right)\right\|_{0, \Omega_{i}} \lesssim h^{\frac{1}{2}}\|\operatorname{curl} \mathbf{w}(\Phi)\|_{\frac{1}{2}, \Omega_{i}} .
$$

This implies

$$
\begin{aligned}
& \left\|\mathbf{r}_{h} \mathbf{w}(\Phi)\right\|_{0, \Omega_{i}}+\left\|\operatorname{curl}\left(\mathbf{r}_{h} \mathbf{w}(\Phi)\right)\right\|_{0, \Omega_{i}} \\
\lesssim & \|\mathbf{w}(\Phi)\|_{0, \Omega_{i}}+\|\operatorname{curl} \mathbf{w}(\Phi)\|_{0, \Omega_{i}}+h^{\frac{1}{2}}\|\operatorname{curl} \mathbf{w}(\Phi)\|_{\frac{1}{2}, \Omega_{i}} .
\end{aligned}
$$

Using (4.29), we see that

$$
\|\mathbf{w}(\Phi)\|_{0, \Omega_{i}}+\|\operatorname{curl} \mathbf{w}(\Phi)\|_{0, \Omega_{i}} \leq\|\tilde{\mathbf{w}}(\Phi)\|_{0, \Omega_{i}}+\|\operatorname{curl} \tilde{\mathbf{w}}(\Phi)\|_{0, \Omega_{i}},
$$

which, together with (4.25) and (4.24), yields

$$
\|\mathbf{w}(\Phi)\|_{0, \Omega_{i}}+\| \text { curl w }(\Phi)\left\|_{0, \Omega_{i}} \lesssim\right\| \Phi\left\|_{-\frac{1}{2}, \Gamma_{i}}+\right\| \operatorname{div}_{\tau} \Phi \|_{-\frac{1}{2}, \Gamma_{i}} .
$$

By (4.20), (4.29) and Lemma 4.8)(4),(3)], we derive

$$
\begin{aligned}
\|\operatorname{curl} \mathbf{w}(\Phi)\|_{\frac{1}{2}, \Omega_{i}} & =\|\operatorname{curl} \tilde{\mathbf{w}}(\Phi)\|_{0, \Omega_{i}}+|\operatorname{curl} \tilde{\mathbf{w}}(\Phi)|_{\frac{1}{2}, \Omega_{i}} \\
& \lesssim d^{-\frac{1}{2}}\|\operatorname{curl} \tilde{\mathbf{w}}(\Phi)\|_{0, \Omega_{i}}+\|\operatorname{curl} \tilde{\mathbf{w}}(\Phi) \cdot \mathbf{n}\|_{0, \Gamma_{i}} \\
& \lesssim d^{-\frac{1}{2}}\left\|\operatorname{div}_{\tau} \Phi\right\|_{-\frac{1}{2}, \Gamma_{i}}+\left\|\operatorname{div}_{\tau} \Phi\right\|_{0, \Gamma_{i}} .
\end{aligned}
$$

Substituting this inequality and (4.31) into (4.30), gives

$\left\|\mathbf{r}_{h} \mathbf{w}(\Phi)\right\|_{0, \Omega_{i}}+\left\|\operatorname{curl}\left(\mathbf{r}_{h} \mathbf{w}(\Phi)\right)\right\|_{0, \Omega_{i}} \lesssim\|\Phi\|_{-\frac{1}{2}, \Gamma_{i}}+\left\|\operatorname{div}_{\tau} \Phi\right\|_{-\frac{1}{2}, \Gamma_{i}}+h^{\frac{1}{2}}\left\|\operatorname{div}_{\tau} \Phi\right\|_{0, \Gamma_{i}}$.

This, together with (4.26), leads to

$\left\|\mathbf{r}_{h} \mathbf{w}(\Phi)\right\|_{0, \Omega_{i}}+\left\|\operatorname{curl}\left(\mathbf{r}_{h} \mathbf{w}(\Phi)\right)\right\|_{0, \Omega_{i}} \lesssim h^{\frac{1}{2}}[1+\log (d / h)]^{\frac{1}{2}}\left(\|\Phi\|_{0, \Gamma_{i}}+\left\|\operatorname{div}_{\tau} \Phi\right\|_{0, \Gamma_{i}}\right)$.

On the other hand, it follows by Lemma $4.8(2)$ that $\left.(\tilde{\mathbf{w}}(\Phi) \cdot \mathbf{n})\right|_{\Gamma_{i}} \in H^{\delta}\left(\Gamma_{i}\right)$. Thus, from the definition of $p(\Phi)$ we infer that $p(\Phi) \in H^{\frac{3}{2}+\delta}\left(\Omega_{i}\right)$ and $\nabla p(\Phi) \in$ $H^{\frac{1}{2}+\delta}\left(\operatorname{curl} ; \Omega_{i}\right)$. Let $\pi_{h}: C\left(\Omega_{i}\right) \rightarrow Z_{h}\left(\Omega_{i}\right)$ be the nodal interpolation operator associated with $\mathcal{T}_{h}$. Then, $\mathbf{r}_{h}(\nabla p(\Phi))=\nabla\left(\pi_{h} p(\Phi)\right)$, and so

$$
\begin{aligned}
& \left\|\mathbf{r}_{h}(\nabla p(\Phi))-\nabla p(\Phi)\right\|_{0, \Omega_{i}} \lesssim h^{\frac{1}{2}}\|p(\Phi)\|_{\frac{3}{2}, \Omega_{i}} \\
\lesssim \quad & h^{\frac{1}{2}}\left(\|\triangle p(\Phi)\|_{0, \Omega_{i}}+\left\|\frac{\partial p(\Phi)}{\partial \mathbf{n}}\right\|_{0, \Gamma_{i}}\right)=h^{\frac{1}{2}}\|\tilde{\mathbf{w}}(\Phi) \cdot \mathbf{n}\|_{0, \Gamma_{i}} .
\end{aligned}
$$

Hence,

$$
\left\|\mathbf{r}_{h}(\nabla p(\Phi))\right\|_{0, \Omega_{i}} \lesssim\|\nabla p(\Phi)\|_{0, \Omega_{i}}+h^{\frac{1}{2}}\|\tilde{\mathbf{w}}(\Phi) \cdot \mathbf{n}\|_{0, \Gamma_{i}}
$$


By (4.29) and (4.24), we have

$$
\|\nabla p(\Phi)\|_{0, \Omega_{i}} \leq\|\tilde{\mathbf{w}}(\Phi)\|_{0, \Omega_{i}} \lesssim\|\Phi\|_{-\frac{1}{2}, \Gamma_{i}}+\left\|\operatorname{div}_{\tau} \Phi\right\|_{-\frac{1}{2}, \Gamma_{i}} .
$$

Then it follows by (4.21), Lemma 4.8(5),(1)] and (4.24)-(4.25) that

$$
\begin{aligned}
\|\tilde{\mathbf{w}}(\Phi) \cdot \mathbf{n}\|_{0, \Gamma_{i}} & \lesssim d^{-\frac{1}{2}}\|\tilde{\mathbf{w}}(\Phi)\|_{0, \Omega_{i}}+d^{\frac{1}{2}}\|\mathbf{c u r l} \tilde{\mathbf{w}}(\Phi)\|_{0, \Omega_{i}}+\|\tilde{\mathbf{w}}(\Phi) \times \mathbf{n}\|_{0, \Gamma_{i}} \\
& \lesssim d^{-\frac{1}{2}}\left(\|\Phi\|_{-\frac{1}{2}, \Gamma_{i}}+\left\|\operatorname{div}_{\tau} \Phi\right\|_{-\frac{1}{2}, \Gamma_{i}}\right)+\|\Phi\|_{0, \Gamma_{i}} .
\end{aligned}
$$

Plugging this and (4.34) into (4.33) and using (4.26) yield

$$
\left\|\mathbf{r}_{h}(\nabla p(\Phi))\right\|_{0, \Omega_{i}} \lesssim h^{\frac{1}{2}}[1+\log (d / h)]^{\frac{1}{2}}\left(\|\Phi\|_{0, \Gamma_{i}}+\left\|\operatorname{div}_{\tau} \Phi\right\|_{0, \Gamma_{i}}\right) .
$$

This, together with (4.29), (4.32) and the fact that $\operatorname{curl}\left(\mathbf{r}_{h} \nabla p(\Phi)\right)=0$, gives (4.28).

4.3. Some estimates with the norms $\|\cdot\|_{1 / 2, \Gamma_{i}},\|\cdot\|_{-1 / 2, \Gamma_{i}}$ and $\|\cdot\|_{*, \mathbf{F}_{b}}$. This section summarizes the results which will be used in the condition number estimates in Section 5. Detailed proofs are omitted here but can be found in [19] and [20].

For any subdomain $\Omega_{i}$, by $\mathcal{W}_{i}$ we denote the set of the edges of $\Omega_{i}$, which also belong to at least two other local interfaces $\Gamma_{j}, j \neq i$. For any given subset $G$ of $\Gamma_{i}$ and a function $\varphi \in L^{2}(G)$, we use $\gamma_{G}(\varphi)$ to denote the average value of $\varphi$ on $G$. For any $\varphi \in Z_{h}\left(\Gamma_{i}\right)$, we define $\pi_{0}^{i} \varphi \in Z_{h}\left(\Gamma_{i}\right)$ as follows:

$$
\pi_{0}^{i} \varphi(\mathbf{x})= \begin{cases}\varphi(\mathbf{x}), & \text { for } \mathbf{x} \in \mathcal{W}_{i} \cap \mathcal{N}_{h}, \\ \gamma_{\mathrm{F}}(\varphi), & \text { for } \mathbf{x} \in \mathrm{F} \cap \mathcal{N}_{h}\left(\mathrm{~F} \subset \Gamma_{i}\right) .\end{cases}
$$

It is easy to see that $\mathrm{I}_{\mathrm{F}}^{t}\left(\varphi-\pi_{0}^{i} \varphi\right)=\mathrm{I}_{\mathrm{F}}^{0}\left(\varphi-\gamma_{\mathrm{F}}(\varphi)\right)$ for any $\mathrm{F} \subset \Gamma_{i}$, and we have

Lemma 4.10. For any $\varphi \in Z_{h}\left(\Gamma_{i}\right)$ and any $\mathrm{F} \subset \Gamma_{i}$, we have (cf. [4] [32] [19])

$$
\begin{aligned}
\|\varphi\|_{0, \partial \mathrm{F}} & \lesssim[1+\log (d / h)]^{\frac{1}{2}}\|\varphi\|_{\frac{1}{2}, \Gamma_{i}}, \\
\left\|\mathrm{I}_{\mathrm{F}}^{0} \varphi\right\|_{\frac{1}{2}, \Gamma_{i}} & \lesssim[1+\log (d / h)]\|\varphi\|_{\frac{1}{2}, \Gamma_{i}}, \\
\left|\mathrm{I}_{\partial \mathrm{F}}^{0} \varphi\right|_{\frac{1}{2}, \mathrm{~F}} & \lesssim[1+\log (d / h)]^{\frac{1}{2}}\|\varphi\|_{\frac{1}{2}, \Gamma_{i}}, \\
\left|\mathrm{I}_{\mathrm{F}}^{t}\left(\varphi-\pi_{0}^{i} \varphi\right)\right|_{\frac{1}{2}, \Gamma_{i}} & \lesssim[1+\log (d / h)]|\varphi|_{\frac{1}{2}, \Gamma_{i}} .
\end{aligned}
$$

For any face $\mathrm{F}$ of $\Gamma_{i}$, we introduce a quantity (not a norm) on $\mathrm{F}_{b}$ as follows:

$$
\|\Phi\|_{*}, \mathrm{~F}_{b}=\left\{\sum_{e \in \mathrm{F}_{b}}\|\mathbf{v}\|_{0, e}^{2}\right\}^{\frac{1}{2}}, \quad \forall \Phi=\left.(\mathbf{v} \times \mathbf{n})\right|_{\Gamma_{i}} \in V_{h}\left(\Gamma_{i}\right) .
$$

Lemma 4.11. Let $\mathrm{F}$ be a given face of $\Omega_{i}$. Then for any $\mathbf{v}_{h} \in Z_{h}\left(\Omega_{i}\right)^{3}$ we have

$$
\left\|\mathbf{v}_{h} \times \mathbf{n}\right\|_{*}, \mathrm{~F}_{b} \lesssim[1+\log (d / h)]^{\frac{1}{2}}\left\|\mathbf{v}_{h}\right\|_{\frac{1}{2}, \Gamma_{i}},
$$

while for any $\mathbf{v}_{h} \in V_{h}\left(\Omega_{i}\right)$ with $\mathbf{w}$ defined as in (4.6) we have

$$
\begin{aligned}
\left\|\left(\mathbf{r}_{h} \mathbf{w}\right) \times \mathbf{n}\right\|_{*}, \mathrm{~F}_{b} & \lesssim[1+\log (d / h)]^{\frac{1}{2}} \| \text { curl } \mathbf{v}_{h} \|_{0, \Omega_{i}}, \\
d^{-2}\left\|\mathbf{r}_{h} \mathbf{w}\right\|_{0, \Omega_{i}}^{2} & \lesssim[1+\log (d / h)] \mid \| \text { curl } \mathbf{v}_{h} \|_{0, \Omega_{i}}^{2} .
\end{aligned}
$$


Lemma 4.12. For any $\Phi \in V_{h}\left(\Gamma_{i}\right)$ and any face $\mathrm{F}$ of $\Gamma_{i}$, we have (cf. 2] [19])

$$
\begin{aligned}
\|\Phi\|_{0, \Gamma_{i}} & \lesssim h^{-\frac{1}{2}}\|\Phi\|_{-\frac{1}{2}, \Gamma_{i}}, \quad\left\|\mathbf{I}_{\mathrm{F}_{b}}^{0} \Phi\right\|_{0, \mathrm{~F}} \lesssim h^{\frac{1}{2}}\|\Phi\|_{*}, \mathrm{~F}_{b}, \\
\left\|\mathbf{I}_{\mathrm{F}_{\partial}}^{0} \Phi\right\|_{-\frac{1}{2}, \Gamma_{i}} & \lesssim[1+\log (d / h)]\|\Phi\|_{-\frac{1}{2}, \Gamma_{i}}+h^{\frac{1}{2}}\|\Phi\|_{*}, \mathrm{~F}_{b}, \\
\left\|\mathbf{I}_{\mathrm{F}_{b}}^{0} \Phi\right\|_{-\frac{1}{2}, \mathrm{~F}} & \lesssim h^{\frac{1}{2}}[1+\log (d / h)]^{\frac{1}{2}}\left\|\mathbf{I}_{\mathrm{F}_{b}}^{0} \Phi\right\|_{0, \mathrm{~F}} .
\end{aligned}
$$

Lemma 4.13. Let $\varphi \in L^{2}\left(\Gamma_{i}\right)$ be piecewise constant with respect to the $\mathcal{T}_{h}$-induced triangulation $\mathcal{T}_{h, i}$ on $\Gamma_{i}$. Then we have $\|\varphi\|_{0, \Gamma_{i}} \lesssim h^{-\frac{1}{2}}\|\varphi\|_{-\frac{1}{2}, \Gamma_{i}}$.

Lemma 4.14. For any $\Phi=\mathbf{v} \times \mathbf{n} \in V_{h}\left(\Gamma_{i}\right)$, we have

$$
\left\|\operatorname{div}_{\tau}\left(\mathbf{I}_{\mathrm{F}}^{0} \Phi\right)\right\|_{-\frac{1}{2}, \Gamma_{i}} \lesssim[1+\log (d / h)]\left\|\operatorname{div}_{\tau} \Phi\right\|_{-\frac{1}{2}, \Gamma_{i}}+[1+\log (d / h)]^{\frac{1}{2}}\|\Phi\|_{*, \mathrm{~F}_{b}},
$$

while for any $\mathbf{v}_{0} \in V^{0}(\Omega)$, let $\Phi_{0}^{i}=\left.\left(\mathbf{v}_{0} \times \mathbf{n}\right)\right|_{\Gamma_{i}} \in V_{h}\left(\Gamma_{i}\right)$. Then we have

$$
\left\|\Phi_{0}^{i}\right\|_{-\frac{1}{2}, \Gamma_{i}}+\left\|\operatorname{div}_{\tau} \Phi_{0}^{i}\right\|_{-\frac{1}{2}, \Gamma_{i}} \lesssim h^{\frac{1}{2}}[1+\log (d / h)]^{\frac{1}{2}}\left(\left\|\Phi_{0}^{i}\right\|_{0, \Gamma_{i}}+\left\|\operatorname{div}_{\tau} \Phi_{0}^{i}\right\|_{0, \Gamma_{i}}\right) .
$$

The estimate in the following lemma indicates that the norm $\left\|\mathbf{I}_{\mathrm{F}}^{0} \Phi\right\|_{\mathcal{X}_{\Gamma_{i}}}$ cannot be bounded by $\|\Phi\|_{\mathcal{X}_{\Gamma_{i}}}$ only (compare with the estimate 4.37)).

Lemma 4.15. Let $\mathbf{w}$ and $\mathbf{v}_{h}$ be the same as specified in Lemma 4.3, and let $\Phi=$ $\mathbf{r}_{h} \mathbf{w} \times \mathbf{n}$ on $\Gamma_{i}$. Then, for any face $\mathrm{F} \subset \Gamma_{i}$ we have

$$
\left\|\mathbf{I}_{\mathrm{F}_{\partial}}^{0} \Phi\right\|_{\mathcal{X}_{\Gamma_{i}}} \lesssim[1+\log (d / h)]\left(\|\Phi\|_{\mathcal{X}_{\Gamma_{i}}}+\| \text { curl } \mathbf{v}_{h} \|_{0, \Omega_{i}}\right)
$$

We end this section with some relation between the edge element space $V_{h}\left(\Omega_{i}\right)$ and the nodal element space $Z_{h}\left(\Omega_{i}\right)$ :

Lemma 4.16. Let $t$ be an edge of $\Omega_{i}$. For any $q \in Z_{h}\left(\Omega_{i}\right)$, if it vanishes on $t$, then its gradient $\nabla q \in V_{h}\left(\Omega_{i}\right)$ and $\lambda_{e}(\nabla q)=0$ for any fine edge $e \subset \mathcal{E}_{h} \cap t$.

\section{Proofs of the main Results}

5.1. Proof of Theorem 3.1. For any $\mathbf{v}_{h} \in V_{h}(\Omega)$, we first decompose it as follows:

$$
\mathbf{v}_{h}=\nabla q_{h} \oplus \mathbf{w}_{h},
$$

where $q_{h} \in Z_{h}(\Omega)$ solves

$$
\left(\beta \nabla q_{h}, \nabla \psi_{h}\right)=\left(\beta \mathbf{v}_{h}, \nabla \psi_{h}\right), \quad \forall \psi_{h} \in Z_{h}(\Omega)
$$

and $\mathbf{w}_{h}$ is orthogonal to $\nabla q_{h}$ in the scalar product $(\beta \cdot, \cdot)$. By Cauchy-Schwarz inequality,

$$
\left\|\beta^{\frac{1}{2}} \nabla q_{h}\right\|_{0, \Omega} \leq\left\|\beta^{\frac{1}{2}} \mathbf{v}_{h}\right\|_{0, \Omega} .
$$

Moreover, we apply Lemma 4.2 for $\mathbf{w}_{h}$ to obtain

$$
\left\|\beta^{\frac{1}{2}} \mathbf{w}_{h}\right\|_{0, \Omega} \lesssim\left\|\beta^{\frac{1}{2}} \operatorname{curl} \mathbf{w}_{h}\right\|_{0, \Omega}=\left\|\beta^{\frac{1}{2}} \operatorname{curl} \mathbf{v}_{h}\right\|_{0, \Omega} .
$$

Let $J: Z_{h}(\Omega) \rightarrow Z_{h}(\Omega)$ be the operator defined by

$$
\left(J \phi_{h}, \psi_{h}\right)=\left(\beta \nabla \phi_{h}, \nabla \psi_{h}\right), \quad \forall \phi, \psi \in Z_{h}(\Omega) .
$$

Then, by the definitions of $q_{h}, B^{t}$ and $J$, we have

$$
\begin{aligned}
\left(\beta \nabla q_{h}, \nabla q_{h}\right) & =\left(\beta \mathbf{v}_{h}, \nabla q_{h}\right)=\left(B^{t} \mathbf{v}_{h}, q_{h}\right) \\
& =\left(J q_{h}, J^{-1} B^{t} \mathbf{v}_{h}\right)=\left(\beta \nabla q_{h}, \nabla\left(J^{-1} B^{t} \mathbf{v}_{h}\right)\right) \\
& =\left(\beta \mathbf{v}_{h}, \nabla\left(J^{-1} B^{t} \mathbf{v}_{h}\right)\right)=\left(B^{t} \mathbf{v}_{h}, J^{-1} B^{t} \mathbf{v}_{h}\right) .
\end{aligned}
$$


Therefore, we derive

$$
\left(B J^{-1} B^{t} \mathbf{v}_{h}, \mathbf{v}_{h}\right)=\left(\beta \nabla q_{h}, \nabla q_{h}\right) .
$$

This, together with (5.2), leads to

$$
\left(B J^{-1} B^{t} \mathbf{v}_{h}, \mathbf{v}_{h}\right) \leq\left(\beta \mathbf{v}_{h}, \mathbf{v}_{h}\right) .
$$

Now it follows from (3.3) that

$$
\begin{aligned}
\left(A \mathbf{v}_{h}, \mathbf{v}_{h}\right) & \lesssim\left(\alpha \operatorname{curl} \mathbf{v}_{h}, \operatorname{curl} \mathbf{v}_{h}\right)+r_{0}\left(B J^{-1} B^{t} \mathbf{v}_{h}, \mathbf{v}_{h}\right) \\
& \leq\left(\alpha \operatorname{curl} \mathbf{v}_{h}, \operatorname{curl} \mathbf{v}_{h}\right)+r_{0}\left(\beta \mathbf{v}_{h}, \mathbf{v}_{h}\right) \\
& \lesssim\left(\tilde{A} \mathbf{v}_{h}, \mathbf{v}_{h}\right) .
\end{aligned}
$$

On the other hand, using (5.5), (5.3) and (3.3) yields

$$
\begin{aligned}
\left(\alpha \mathbf{v}_{h}, \mathbf{v}_{h}\right) & =r_{0}\left(\beta \mathbf{v}_{h}, \mathbf{v}_{h}\right)=r_{0}\left[\left(\beta \nabla q_{h}, \nabla q_{h}\right)+\left(\beta \mathbf{w}_{h}, \mathbf{w}_{h}\right)\right] \\
& \lesssim r_{0}\left[\left(B J^{-1} B^{t} \mathbf{v}_{h}, \mathbf{v}_{h}\right)+\left(\beta \operatorname{curl} \mathbf{v}_{h}, \operatorname{curl} \mathbf{v}_{h}\right)\right] \\
& \lesssim \max \{1, G(d / h)\}\left(A \mathbf{v}_{h}, \mathbf{v}_{h}\right) \lesssim G(d / h)\left(A \mathbf{v}_{h}, \mathbf{v}_{h}\right),
\end{aligned}
$$

and so

$$
\left(\tilde{A} \mathbf{v}_{h}, \mathbf{v}_{h}\right) \lesssim G(d / h)\left(A \mathbf{v}_{h}, \mathbf{v}_{h}\right) .
$$

This, together with (5.6), gives the desired result.

5.2. Proof of Theorem 3.2. This subsection is devoted to the estimate of the condition number of the preconditioned system $\hat{A}^{-1} A$; see (3.7). The following lemma reduces this task to the estimates of two positive constants $C_{1}$ and $C_{2}$. This framework can be regarded as a variant of the additive Schwarz theory associated with the space decomposition (3.5) (refer to [27] and 31]), and the proof is standard (cf. [15] and [28]). Here, we have used the orthogonality between $V^{p}(\Omega)$ and $V^{H}(\Omega)=V^{0}(\Omega)+\sum_{\Gamma_{i j}} V^{i j}(\Omega)$.

Lemma 5.1. Assume that the following two conditions hold:

(i) For any $\mathbf{v} \in V^{H}(\Omega)$ there is a decomposition

$$
\mathbf{v}=\mathbf{v}_{0}+\sum_{\Gamma_{i j}} \mathbf{v}_{i j}
$$

$$
\text { with } \mathbf{v}_{0} \in V^{0}(\Omega) \text { and } \mathbf{v}_{i j} \in V^{i j}(\Omega) \text {, such that }
$$

$$
\left(\hat{A}_{0} \mathbf{v}_{0}, \mathbf{v}_{0}\right)+\sum_{\Gamma_{i j}}\left(\hat{A}_{i j} \mathbf{v}_{i j}, \mathbf{v}_{i j}\right) \leq C_{1}(\tilde{A} \mathbf{v}, \mathbf{v}) .
$$

(ii) For any $\mathbf{w}_{0} \in V^{0}(\Omega)$ and $\mathbf{w}_{i j} \in V^{i j}(\Omega)$, we have

$$
\left(\tilde{A}\left(\mathbf{w}_{0}+\sum_{\Gamma_{i j}} \mathbf{w}_{i j}\right), \mathbf{w}_{0}+\sum_{\Gamma_{i j}} \mathbf{w}_{i j}\right) \leq C_{2}\left\{\left(\hat{A}_{0} \mathbf{w}_{0}, \mathbf{w}_{0}\right)+\sum_{\Gamma_{i j}}\left(\hat{A}_{i j} \mathbf{w}_{i j}, \mathbf{w}_{i j}\right)\right\} .
$$

Then we have the following estimate

$$
\operatorname{cond}\left(\hat{A}^{-1} \tilde{A}\right) \leq C_{1} C_{2} \text {. }
$$

By this lemma and Theorem 3.1, we obtain

$$
\operatorname{cond}\left(\hat{A}^{-1} A\right) \leq C_{1} C_{2} G(d / h) \text {. }
$$

For the proof of Theorem 3.2, it suffices to estimate the constants $C_{1}$ and $C_{2}$ in Lemma 5.1 
To do so, we first introduce some notation. For any $\mathbf{v}_{h} \in V^{H}(\Omega)$, let $\mathbf{v}_{h}^{i}=\left.\mathbf{v}_{h}\right|_{\Omega_{i}}$. From the discussions in Subsection 4.1, there exist $p^{i} \in H^{1}\left(\Omega_{i}\right), p_{h}^{i} \in Z_{h}\left(\Omega_{i}\right)$ and $\mathbf{w}^{i} \in H^{1}\left(\Omega_{i}\right)^{3}$ such that

$$
\begin{gathered}
\mathbf{v}_{h}^{i}=\nabla p^{i} \oplus \mathbf{w}^{i}, \\
\mathbf{v}_{h}^{i}=\nabla p_{h}^{i}+\mathbf{r}_{h} \mathbf{w}^{i}=\nabla p_{h}^{i}+\mathbf{w}_{h}^{i}
\end{gathered}
$$

with $\mathbf{w}_{h}^{i}=\mathbf{r}_{h} \mathbf{w}^{i} \in V_{h}\left(\Omega_{i}\right)$. By (5.9) and (5.10), we know

$$
\operatorname{curl} \mathbf{w}_{h}^{i}=\operatorname{curl} \mathbf{w}^{i}=\operatorname{curl} \mathbf{v}_{h}^{i} .
$$

We are now ready to show Theorem 3.2 using Lemma 5.1 and to divide the proof into four steps.

Step 1: Establish a suitable decomposition for $\mathbf{v}_{h} \in V^{H}(\Omega)$.

We introduce $p_{h 0}^{i} \in Z_{h}\left(\Omega_{i}\right)$ and $\mathbf{w}_{h 0}^{i} \in V_{h}\left(\Omega_{i}\right)$ by

$$
p_{h 0}^{i}=R_{h}^{i} \pi_{0}^{i}\left(\left.p_{h}^{i}\right|_{\Gamma_{i}}\right), \quad \mathbf{w}_{h 0}^{i}=\left.\mathbf{R}_{h}^{i} \mathbf{I}_{\Delta_{i}}^{0}\left(\mathbf{w}_{h}^{i} \times \mathbf{n}\right)\right|_{\Gamma_{i}}, \quad i=1, \cdots, N .
$$

Define

and

$$
\Phi_{0}^{i}=\left(\nabla p_{h 0}^{i}+\mathbf{w}_{h 0}^{i}\right) \times\left.\mathbf{n}\right|_{\Gamma_{i}}
$$

$$
\Phi_{0}=\frac{\sqrt{\alpha_{i}}}{\sqrt{\alpha_{i}}+\sqrt{\alpha_{j}}} \Phi_{0}^{i}+\frac{\sqrt{\alpha_{j}}}{\sqrt{\alpha_{i}}+\sqrt{\alpha_{j}}} \Phi_{0}^{j} \quad \text { on } \Gamma_{i j} \subset \Gamma \text {. }
$$

Set $\Phi=\left.\left(\mathbf{v}_{h} \times \mathbf{n}\right)\right|_{\Gamma}$. It follows from Lemma 4.16 that $\left.\left(\Phi_{0}-\Phi\right)\right|_{\mathcal{W}_{i}}=0$ for every $i$. Thus, $\Phi_{0}$ is defined uniquely on all the edges of the interface $\Gamma$. In particular, $\Phi_{0}$ equals $\Phi$ on these edges. Define $\mathbf{v}_{0} \in V^{0}(\Omega)$ such that $\left.\mathbf{v}_{0}\right|_{\Omega_{i}}$ is the discrete $A_{i}$-extension of $\left.\Phi_{0}\right|_{\Gamma_{i}}$. For each $\Gamma_{i j} \subset \Gamma$ define $\mathbf{v}_{i j} \in V_{i j}(\Omega)$, such that

$$
\left.\left(\mathbf{v}_{i j} \times \mathbf{n}\right)\right|_{\Gamma_{i j}}=\left(\mathbf{v}_{h}-\mathbf{v}_{0}\right) \times\left.\mathbf{n}\right|_{\Gamma_{i j}} .
$$

It is easy to see that

$$
\mathbf{v}_{h}=\mathbf{v}_{0}+\sum_{\Gamma_{i j}} \mathbf{v}_{i j} \quad \text { in } \Omega .
$$

Step 2: Derive the estimate

$$
\left(\hat{A}_{0} \mathbf{v}_{0}, \mathbf{v}_{0}\right) \lesssim[1+\log (d / h)]^{2}\left(\tilde{A} \mathbf{v}_{h}, \mathbf{v}_{h}\right)
$$

We first estimate the term $h[1+\log (d / h)] \sum_{i=1}^{N} \alpha_{i}\left\|\left.\operatorname{div}_{\tau}\left(\mathbf{v}_{0} \times \mathbf{n}\right)\right|_{\Gamma_{i}}\right\|_{0, \Delta_{i}}^{2}$.

By Lemma 6.2 in the Appendix and by the triangle inequality, we obtain for $\mathrm{F}=\Gamma_{i} \cap \Gamma_{j}$ that

$$
\begin{aligned}
& \left\|\left.\operatorname{div}_{\tau}\left(\mathbf{v}_{0} \times \mathbf{n}\right)\right|_{\Gamma_{i}}\right\|_{0, \mathrm{~F}_{b}} \lesssim\left\|\left.\operatorname{div}_{\tau}\left(\mathbf{v}_{h}^{i} \times \mathbf{n}\right)\right|_{\Gamma_{i}}\right\|_{0, \mathrm{~F}_{b}} \\
& +\left\|\left.\frac{\sqrt{\alpha_{i}}}{\sqrt{\alpha_{i}}+\sqrt{\alpha_{j}}} \operatorname{div}_{\tau} \mathbf{I}_{\mathrm{F}_{\partial}}^{0}\left(\mathbf{w}_{h}^{i} \times \mathbf{n}\right)\right|_{\Gamma_{i}}+\left.\frac{\sqrt{\alpha_{i}}}{\sqrt{\alpha_{i}}+\sqrt{\alpha_{j}}} \operatorname{div}_{\tau} \mathbf{I}_{\mathrm{F}_{\partial}}^{0}\left(\mathbf{w}_{h}^{j} \times \mathbf{n}\right)\right|_{\Gamma_{j}}\right\|_{0, \mathrm{~F}_{b}} \\
& \lesssim\left\|\left.\operatorname{div}_{\tau}\left(\mathbf{v}_{h}^{i} \times \mathbf{n}\right)\right|_{\Gamma_{i}}\right\|_{0, \Gamma_{i}}+\frac{\sqrt{\alpha_{i}}}{\sqrt{\alpha_{i}}+\sqrt{\alpha_{j}}}\left\|\left.\operatorname{div}_{\tau} \mathbf{I}_{\mathrm{F}_{\partial}}^{0}\left(\mathbf{w}_{h}^{i} \times \mathbf{n}\right)\right|_{\Gamma_{i}}\right\|_{0, \mathrm{~F}_{b}} \\
& +\frac{\sqrt{\alpha_{j}}}{\sqrt{\alpha_{i}}+\sqrt{\alpha_{j}}}\left\|\left.\operatorname{div}_{\tau} \mathbf{I}_{\mathrm{F}_{\partial}}^{0}\left(\mathbf{w}_{h}^{j} \times \mathbf{n}\right)\right|_{\Gamma_{j}}\right\|_{0, \mathrm{~F}_{b}} .
\end{aligned}
$$

By Lemma 4.13 and the inequality (2.11) in [1, we have

$$
\left\|\left.\operatorname{div}_{\tau}\left(\mathbf{v}_{h}^{i} \times \mathbf{n}\right)\right|_{\Gamma_{i}}\right\|_{0, \Gamma_{i}} \lesssim h^{-\frac{1}{2}}\left\|\left.\operatorname{div}_{\tau}\left(\mathbf{v}_{h}^{i} \times \mathbf{n}\right)\right|_{\Gamma_{i}}\right\|_{-\frac{1}{2}, \Gamma_{i}} \lesssim h^{-\frac{1}{2}}\left\|\operatorname{curl} \mathbf{v}_{h}^{i}\right\|_{0, \Omega_{i}} .
$$


Moreover, similarly to the proof of Lemma 4.14 (see [19]), one can show

$$
\begin{aligned}
\left\|\left.\operatorname{div}_{\tau} \mathbf{I}_{\mathrm{F}_{\partial}}^{0}\left(\mathbf{w}_{h}^{i} \times \mathbf{n}\right)\right|_{\Gamma_{i}}\right\|_{0, \mathrm{~F}_{b}} & \lesssim h^{-\frac{1}{2}}\left\|\mathbf{w}_{h}^{i} \times \mathbf{n}\right\|_{*, \mathrm{~F}_{b}}, \\
\left\|\left.\operatorname{div}_{\tau} \mathbf{I}_{\mathrm{F}_{\partial}}^{0}\left(\mathbf{w}_{h}^{j} \times \mathbf{n}\right)\right|_{\Gamma_{i}}\right\|_{0, \mathrm{~F}_{b}} & \lesssim h^{-\frac{1}{2}}\left\|\mathbf{w}_{h}^{j} \times \mathbf{n}\right\|_{*, \mathrm{~F}_{b}} .
\end{aligned}
$$

Substituting these two estimates and (5.14) into (5.13) and using Lemma 4.11lead to

$$
\begin{aligned}
& h\left\|\left.\operatorname{div}_{\tau}\left(\mathbf{v}_{0} \times \mathbf{n}\right)\right|_{\Gamma_{i}}\right\|_{0, \mathrm{~F}_{b}}^{2} \lesssim\left\|\operatorname{curl}_{\mathbf{h}}^{i}\right\|_{0, \Omega_{i}}^{2} \\
& \quad+[1+\log (d / h)]\left\{\left(\frac{\sqrt{\alpha_{i}}}{\sqrt{\alpha_{i}}+\sqrt{\alpha_{j}}}\right)^{2}\left\|\operatorname{curl} \mathbf{v}_{h}^{i}\right\|_{0, \Omega_{i}}^{2}+\left(\frac{\sqrt{\alpha_{j}}}{\sqrt{\alpha_{i}}+\sqrt{\alpha_{j}}}\right)^{2}\left\|\operatorname{curl} \mathbf{v}_{h}^{j}\right\|_{0, \Omega_{j}}^{2}\right\} .
\end{aligned}
$$

A similar estimate holds on $\Gamma_{j}$ as well. Then using $\alpha_{i}+\alpha_{j}<\left(\sqrt{\alpha_{i}}+\sqrt{\alpha_{j}}\right)^{2}$, we derive

$$
\begin{aligned}
& h\left\{\alpha_{i}\left\|\left.\operatorname{div}_{\tau}\left(\mathbf{v}_{0} \times \mathbf{n}\right)\right|_{\Gamma_{i}}\right\|_{0, \mathrm{~F}_{b}}^{2}+\alpha_{j}\left\|\left.\operatorname{div}_{\tau}\left(\mathbf{v}_{0} \times \mathbf{n}\right)\right|_{\Gamma_{j}}\right\|_{0, \mathrm{~F}_{b}}^{2}\right\} \\
\lesssim & {[1+\log (d / h)]\left\{\alpha_{i}\left\|\operatorname{curl}_{h}^{i}\right\|_{0, \Omega_{i}}^{2}+\alpha_{j}\left\|\operatorname{curl} \mathbf{v}_{h}^{j}\right\|_{0, \Omega_{j}}^{2}\right\} . }
\end{aligned}
$$

This yields

$$
\begin{aligned}
& h[1+\log (d / h)] \sum_{i=1}^{N} \alpha_{i}\left\|\left.\operatorname{div}_{\tau}\left(\mathbf{v}_{0} \times \mathbf{n}\right)\right|_{\Gamma_{i}}\right\|_{0, \Delta_{i}}^{2} \\
& =[1+\log (d / h)] \sum_{\Gamma_{i j} \subset \Gamma} h\left\{\alpha_{i}\left\|\left.\operatorname{div}_{\tau}\left(\mathbf{v}_{0} \times \mathbf{n}\right)\right|_{\Gamma_{i}}\right\|_{0, \mathrm{~F}_{b}}^{2}+\alpha_{j}\left\|\left.\operatorname{div}_{\tau}\left(\mathbf{v}_{0} \times \mathbf{n}\right)\right|_{\Gamma_{j}}\right\|_{0, \mathrm{~F}_{b}}^{2}\right\} \\
& \lesssim[1+\log (d / h)]^{2} \sum_{i=1}^{N} \alpha_{i}\left\|\operatorname{curl} \mathbf{v}_{h}^{i}\right\|_{0, \Omega_{i}}^{2} .
\end{aligned}
$$

Next, we estimate the term $h[1+\log (d / h)] \sum_{i=1}^{N} \alpha_{i}\left\|\mathbf{v}_{0} \times \mathbf{n}\right\|_{0, \Delta_{i}}^{2}$.

From the definition of $\mathbf{v}_{0}$ and the triangle inequality, we have $\left(\mathrm{F}=\Gamma_{i j}\right)$

$$
\begin{aligned}
\left\|\mathbf{v}_{0} \times \mathbf{n}\right\|_{0, \mathrm{~F}_{b}} & \lesssim \frac{\sqrt{\alpha_{i}}}{\sqrt{\alpha_{i}}+\sqrt{\alpha_{j}}}\left(\left\|\nabla p_{h 0}^{i} \times \mathbf{n}\right\|_{0, \mathrm{~F}_{b}}+\left\|\mathbf{w}_{h 0}^{i} \times \mathbf{n}\right\|_{0, \mathrm{~F}_{b}}\right) \\
& +\frac{\sqrt{\alpha_{j}}}{\sqrt{\alpha_{i}}+\sqrt{\alpha_{j}}}\left(\left\|\nabla p_{h 0}^{j} \times \mathbf{n}\right\|_{0, \mathrm{~F}_{b}}+\left\|\mathbf{w}_{h 0}^{j} \times \mathbf{n}\right\|_{0, \mathrm{~F}_{b}}\right) .
\end{aligned}
$$

By Lemma 6.2 (see the Appendix) and (4.36), we can deduce

$$
\begin{aligned}
\left\|\nabla p_{h 0}^{i} \times \mathbf{n}\right\|_{0, \mathrm{~F}_{b}} & \lesssim h^{-\frac{1}{2}}\left\|p_{h}^{i}-\gamma_{\mathrm{F}}\left(p_{h}^{i}\right)\right\|_{0, \partial \mathrm{F}} \\
& \lesssim h^{-\frac{1}{2}}[1+\log (d / h)]^{\frac{1}{2}}\left\|p_{h}^{i}-\gamma_{\mathrm{F}}\left(p_{h}^{i}\right)\right\|_{\frac{1}{2}, \Gamma_{i}} \\
& \lesssim h^{-\frac{1}{2}}[1+\log (d / h)]^{\frac{1}{2}}\left|p_{h}^{i}\right|_{\frac{1}{2}, \Gamma_{i}} \\
& \lesssim h^{-\frac{1}{2}}[1+\log (d / h)]^{\frac{1}{2}}\left|p_{h}^{i}\right|_{1, \Omega_{i} .}
\end{aligned}
$$

Similarly, we have

$$
\left\|\nabla p_{h 0}^{j} \times \mathbf{n}\right\|_{0, \mathrm{~F}_{b}} \lesssim h^{-\frac{1}{2}}[1+\log (d / h)]^{\frac{1}{2}}\left|p_{h}^{j}\right|_{1, \Omega_{j}} .
$$

On the other hand, it follows from (4.43) and Lemma 4.11 that

$$
\left\|\mathbf{w}_{h 0}^{i} \times \mathbf{n}\right\|_{0, \mathrm{~F}_{b}} \lesssim h^{\frac{1}{2}}[1+\log (d / h)]^{\frac{1}{2}}\left\|\mathbf{c u r l} \mathbf{v}_{h}^{i}\right\|_{0, \Omega_{i}}
$$


and

$$
\left\|\mathbf{w}_{h 0}^{j} \times \mathbf{n}\right\|_{0, \mathrm{~F}_{b}} \lesssim h^{\frac{1}{2}}[1+\log (d / h)]^{\frac{1}{2}}\left\|\operatorname{curl} \mathbf{v}_{h}^{j}\right\|_{0, \Omega_{j}} .
$$

Plugging these and (5.17) and (5.18) into (5.16) yields

$$
\begin{aligned}
\left\|\mathbf{v}_{0} \times \mathbf{n}\right\|_{0, \mathrm{~F}_{b}} & \lesssim h^{-\frac{1}{2}}[1+\log (d / h)]^{\frac{1}{2}}\left[\frac{\sqrt{\alpha_{i}}}{\sqrt{\alpha_{i}}+\sqrt{\alpha_{j}}}\left(\left|p_{h}^{i}\right|_{1, \Omega_{i}}+\left\|\operatorname{curl} \mathbf{v}_{h}^{i}\right\|_{0, \Omega_{i}}\right)\right. \\
& \left.+\frac{\sqrt{\alpha_{j}}}{\sqrt{\alpha_{i}}+\sqrt{\alpha_{j}}}\left(\left|p_{h}^{j}\right|_{1, \Omega_{j}}+\left\|\operatorname{curl} \mathbf{v}_{h}^{j}\right\|_{0, \Omega_{j}}\right)\right] .
\end{aligned}
$$

Thus $\left(\mathrm{F}=\Gamma_{i j}\right)$,

$$
\begin{aligned}
h[1+ & \log (d / h)] \sum_{i=1}^{N} \alpha_{i}\left\|\mathbf{v}_{0} \times \mathbf{n}\right\|_{0, \Delta_{i}}^{2}=h[1+\log (d / h)] \sum_{\mathrm{F} \subset \Gamma}\left(\alpha_{i}+\alpha_{j}\right)\left\|\mathbf{v}_{0} \times \mathbf{n}\right\|_{0, \mathrm{~F}_{b}}^{2} \\
\lesssim & {[1+\log (d / h)]^{2} } \\
& \times \sum_{\Gamma_{i j} \subset \Gamma}\left[\alpha_{i}\left(\left|p_{h}^{i}\right|_{1, \Omega_{i}}+\left\|\operatorname{curl} \mathbf{v}_{h}^{i}\right\|_{0, \Omega_{i}}\right)+\alpha_{j}\left(\left|p_{h}^{j}\right|_{1, \Omega_{j}}+\left\|\operatorname{curl}_{\mathbf{v}_{h}^{j}}\right\|_{0, \Omega_{j}}\right)\right] \\
= & {[1+\log (d / h)]^{2} \sum_{i=1}^{N} \alpha_{i}\left(\left|p_{h}^{i}\right|_{1, \Omega_{i}}+\left\|\operatorname{curl} \mathbf{v}_{h}^{i}\right\|_{0, \Omega_{i}}\right) . }
\end{aligned}
$$

This, together with (5.15) and (4.17), leads to (5.12).

Step 3: Prove the estimate

$$
\sum_{\Gamma_{i j}}\left(\hat{A}_{i j} \mathbf{v}_{i j}, \mathbf{v}_{i j}\right) \lesssim[1+\log (d / h)]^{2}\left(\tilde{A} \mathbf{v}_{h}, \mathbf{v}_{h}\right) .
$$

Since $\mathbf{v}_{h}^{i}=\mathbf{v}_{h}^{j}$ on $\Gamma_{i j}$, we have

$$
\mathbf{v}_{h}=\frac{\sqrt{\alpha_{i}}}{\sqrt{\alpha_{i}}+\sqrt{\alpha_{j}}} \mathbf{v}_{h}^{i}+\frac{\sqrt{\alpha_{j}}}{\sqrt{\alpha_{i}}+\sqrt{\alpha_{j}}} \mathbf{v}_{h}^{j} \quad \text { on } \Gamma_{i j} .
$$

Hence, $\mathbf{v}_{i j}$ on $\Gamma_{i j}$ can be written as

$$
\begin{aligned}
\mathbf{v}_{i j}= & \frac{\sqrt{\alpha_{i}}}{\sqrt{\alpha_{i}}+\sqrt{\alpha_{j}}} \nabla\left(p_{h}^{i}-p_{h 0}^{i}\right) \times \mathbf{n}+\frac{\sqrt{\alpha_{j}}}{\sqrt{\alpha_{i}}+\sqrt{\alpha_{j}}} \nabla\left(p_{h}^{j}-p_{h 0}^{j}\right) \times \mathbf{n} \\
& +\frac{\sqrt{\alpha_{i}}}{\sqrt{\alpha_{i}}+\sqrt{\alpha_{j}}}\left(\mathbf{w}_{h}^{i}-\mathbf{w}_{h 0}^{i}\right) \times \mathbf{n}+\frac{\sqrt{\alpha_{j}}}{\sqrt{\alpha_{i}}+\sqrt{\alpha_{j}}}\left(\mathbf{w}_{h}^{j}-\mathbf{w}_{h 0}^{j}\right) \times \mathbf{n} .
\end{aligned}
$$

Define

$$
\begin{aligned}
p_{i j}^{i} & =R_{h}^{i} \mathbf{I}_{i j}^{t}\left\{\left.\frac{\sqrt{\alpha_{i}}}{\sqrt{\alpha_{i}}+\sqrt{\alpha_{j}}}\left(p_{h}^{i}-p_{h 0}^{i}\right)\right|_{\Gamma_{i j}}+\left.\frac{\sqrt{\alpha_{j}}}{\sqrt{\alpha_{i}}+\sqrt{\alpha_{j}}}\left(p_{h}^{j}-p_{h 0}^{j}\right)\right|_{\Gamma_{i j}}\right\} \in Z_{h}\left(\Omega_{i}\right), \\
\mathbf{w}_{i j}^{i} & =\mathbf{R}_{h}^{i} \mathbf{I}_{i j}^{t}\left\{\frac{\sqrt{\alpha_{i}}}{\sqrt{\alpha_{i}}+\sqrt{\alpha_{j}}}\left(\mathbf{w}_{h}^{i}-\mathbf{w}_{h 0}^{i}\right) \times\left.\mathbf{n}\right|_{\Gamma_{i j}}+\frac{\sqrt{\alpha_{j}}}{\sqrt{\alpha_{i}}+\sqrt{\alpha_{j}}}\left(\mathbf{w}_{h}^{j}-\mathbf{w}_{h 0}^{j}\right) \times\left.\mathbf{n}\right|_{\Gamma_{i j}}\right\} \\
\in & \in V_{h}\left(\Omega_{i}\right), \\
\mathbf{v}_{i j}^{i} & =\nabla p_{i j}^{i}+\mathbf{w}_{i j}^{i} \in V_{h}\left(\Omega_{i}\right) .
\end{aligned}
$$

One can verify by Lemma 4.16 that

$$
\mathbf{v}_{i j}^{i} \times \mathbf{n}=\mathbf{v}_{i j} \times \mathbf{n} \quad \text { on } \Gamma_{i} .
$$


Thus, we obtain by the minimum curl-energy property of the discrete $A_{i}$-extension that

$$
\begin{aligned}
\left(A_{i}\left(\left.\mathbf{v}_{i j}\right|_{\Omega_{i}}\right),\left.\mathbf{v}_{i j}\right|_{\Omega_{i}}\right)_{\Omega_{i}} & \leq\left(A_{i} \mathbf{v}_{i j}^{i}, \mathbf{v}_{i j}^{i}\right)=\left\|\alpha^{\frac{1}{2}} \operatorname{curl} \mathbf{w}_{i j}^{i}\right\|_{0, \Omega_{i}}^{2}+\left\|\alpha^{\frac{1}{2}} \mathbf{v}_{i j}^{i}\right\|_{0, \Omega_{i}}^{2} \\
& \lesssim \alpha_{i}\left(\left\|\nabla p_{i j}^{i}\right\|_{0, \Omega_{i}}^{2}+\left\|\operatorname{curl} \mathbf{w}_{i j}^{i}\right\|_{0, \Omega_{i}}^{2}+\left\|\mathbf{w}_{i j}^{i}\right\|_{0, \Omega_{i}}^{2}\right) .
\end{aligned}
$$

As $p_{h 0}^{i}=\pi_{0}^{i}\left(\left.p_{h}^{i}\right|_{\Gamma_{i}}\right)$ on $\Gamma_{i}$, we have

$$
\mathrm{I}_{i j}^{t}\left[\left.\left(p_{h}^{i}-p_{h 0}^{i}\right)\right|_{\Gamma_{i j}}\right]=\mathrm{I}_{i j}^{0}\left(\left.p_{h}^{i}\right|_{\Gamma_{i}}-\pi_{0}^{i}\left(\left.p_{h}^{i}\right|_{\Gamma_{i}}\right)\right) .
$$

Then using (4.39) and the trace theorem, we obtain

$$
\begin{aligned}
& \left\|\nabla p_{i j}^{i}\right\|_{0, \Omega_{i}}^{2}=\left|p_{i j}^{i}\right|_{1, \Omega_{i}}^{2} \lesssim\left|\left(p_{i j}^{i}||_{\Gamma_{i j}}\right)\right|_{\frac{1}{2}, \Gamma_{i}}^{2} \\
& \lesssim\left|\frac{\sqrt{\alpha_{i}}}{\sqrt{\alpha_{i}}+\sqrt{\alpha_{j}}} \mathrm{I}_{i j}^{0}\left(\left.p_{h}^{i}\right|_{\Gamma_{i}}-\pi_{0}^{i}\left(\left.p_{h}^{i}\right|_{\Gamma_{i}}\right)\right)\right|_{\frac{1}{2}, \Gamma_{i}}^{2}+\left|\frac{\sqrt{\alpha_{j}}}{\sqrt{\alpha_{i}}+\sqrt{\alpha_{j}}} \mathrm{I}_{i j}^{0}\left(\left.p_{h}^{j}\right|_{\Gamma_{j}}-\pi_{0}^{j}\left(p_{h}^{j} \mid \Gamma_{\Gamma_{j}}\right)\right)\right|_{\frac{1}{2}, \Gamma_{j}}^{2} \\
& \lesssim[1+\log (d / h)]^{2}\left\{\left(\frac{\sqrt{\alpha_{i}}}{\sqrt{\alpha_{i}}+\sqrt{\alpha_{j}}}\right)^{2}\left|p_{h}^{i}\right|_{\frac{1}{2}, \Gamma_{i}}^{2}+\left(\frac{\sqrt{\alpha_{j}}}{\sqrt{\alpha_{i}}+\sqrt{\alpha_{j}}}\right)^{2}\left|p_{h}^{j}\right|_{\frac{1}{2}, \Gamma_{j}}^{2}\right\} \\
& \lesssim[1+\log (d / h)]^{2}\left\{\left(\frac{\sqrt{\alpha_{i}}}{\sqrt{\alpha_{i}}+\sqrt{\alpha_{j}}}\right)^{2}\left|p_{h}^{i}\right|_{1, \Omega_{i}}^{2}+\left(\frac{\sqrt{\alpha_{j}}}{\sqrt{\alpha_{i}}+\sqrt{\alpha_{j}}}\right)^{2}\left|p_{h}^{j}\right|_{1, \Omega_{j}}^{2}\right\} .
\end{aligned}
$$

We next estimate $\mathbf{w}_{i j}^{i}$. It follows by Lemma 4.6 that

$$
\begin{aligned}
\left\|\operatorname{curl} \mathbf{w}_{i j}^{i}\right\|_{0, \Omega_{i}}^{2}+\left\|\mathbf{w}_{i j}^{i}\right\|_{0, \Omega_{i}}^{2} & \lesssim\left\|\left.\left(\mathbf{w}_{i j}^{i} \times \mathbf{n}\right)\right|_{\Gamma_{i}}\right\|_{\mathcal{X}_{\Gamma_{i}}}^{2} \\
& \lesssim\left\|\frac{\sqrt{\alpha_{i}}}{\sqrt{\alpha_{i}}+\sqrt{\alpha_{j}}} \mathbf{I}_{i j}^{t}\left[\left(\mathbf{w}_{h}^{i}-\mathbf{w}_{h 0}^{i}\right) \times\left.\mathbf{n}\right|_{\Gamma_{i j}}\right]\right\|_{\mathcal{X}_{\Gamma_{i}}}^{2} \\
& +\left\|\frac{\sqrt{\alpha_{j}}}{\sqrt{\alpha_{i}}+\sqrt{\alpha_{j}}} \mathbf{I}_{i j}^{t}\left[\left(\mathbf{w}_{h}^{j}-\mathbf{w}_{h 0}^{j}\right) \times\left.\mathbf{n}\right|_{\Gamma_{i j}}\right]\right\|_{\mathcal{X}_{\Gamma_{j}}}^{2} .
\end{aligned}
$$

For each (open) common face $\mathrm{F}=\Gamma_{i j}$ shared by $\Omega_{i}$ and $\Omega_{j}$, it follows from the definitions of $\mathbf{w}_{h 0}^{i}$ that

$$
\lambda_{e}\left(\mathbf{w}_{h}^{i}-\mathbf{w}_{h 0}^{i}\right)=\left\{\begin{array}{cl}
0, & \text { if } e \subset \mathrm{F}_{b}, \\
\lambda_{e}\left(\mathbf{w}_{h}^{i}\right), & \text { if } e \subset \mathrm{F}_{\partial} .
\end{array}\right.
$$

Then we derive by using Lemma 4.15 that

$$
\begin{aligned}
& \left\|\frac{\sqrt{\alpha_{i}}}{\sqrt{\alpha_{i}}+\sqrt{\alpha_{j}}} \mathbf{I}_{i j}^{t}\left[\left(\mathbf{w}_{h}^{i}-\mathbf{w}_{h 0}^{i}\right) \times\left.\mathbf{n}\right|_{\Gamma_{i j} j}\right]\right\|_{\mathcal{X}_{\Gamma_{i}}}^{2} \\
& \quad=\left(\frac{\sqrt{\alpha_{i}}}{\sqrt{\alpha_{i}}+\sqrt{\alpha_{j}}}\right)^{2}\left\|\mathbf{I}_{\mathrm{F}_{\partial}}^{0}\left[\left.\left(\mathbf{w}_{h}^{i} \times \mathbf{n}\right)\right|_{\Gamma_{i j}}\right]\right\|_{\mathcal{X}_{\Gamma_{i}}}^{2} \\
& \quad \lesssim[1+\log (d / h)]^{2}\left(\frac{\sqrt{\alpha_{i}}}{\sqrt{\alpha_{i}}+\sqrt{\alpha_{j}}}\right)^{2}\left(\left\|\mathbf{w}_{h}^{i} \times \mathbf{n}\right\|_{\mathcal{X}_{\Gamma_{i}}}^{2}+\| \text { curl } \mathbf{v}_{h}^{i} \|_{0, \Omega_{i}}^{2}\right) .
\end{aligned}
$$

On the other hand, for the term $\mathbf{w}_{h}^{i} \times \mathbf{n}$ we have by Lemma 4.5 and (5.9) that

$$
\begin{aligned}
\left\|\mathbf{w}_{h}^{i} \times \mathbf{n}\right\|_{\mathcal{X}_{\Gamma_{i}}}^{2} & \lesssim\left\|\mathbf{w}_{h}^{i}\right\|_{\text {curl } ; \Omega_{i}}^{2} \\
& =\left\|\operatorname{curl}_{h}^{i}\right\|_{0 ; \Omega_{i}}^{2}+d^{-2}\left\|\mathbf{w}_{h}^{i}\right\|_{0 ; \Omega_{i}}^{2} \\
& =\left\|\operatorname{curl} \mathbf{v}_{h}^{i}\right\|_{0 ; \Omega_{i}}^{2}+d^{-2}\left\|\mathbf{w}_{h}^{i}\right\|_{0 ; \Omega_{i}}^{2} .
\end{aligned}
$$


Combining this with (5.23) and using Lemma 4.11 give

$$
\begin{aligned}
& \left\|\frac{\sqrt{\alpha_{i}}}{\sqrt{\alpha_{i}}+\sqrt{\alpha_{j}}} \mathbf{I}_{i j}^{t}\left[\left(\mathbf{w}_{h}^{i}-\mathbf{w}_{h 0}^{i}\right) \times\left.\mathbf{n}\right|_{\Gamma_{i j} j}\right]\right\|_{\mathcal{X}_{\Gamma_{i}}}^{2} \\
& \quad \lesssim[1+\log (d / h)]^{2}\left(\frac{\sqrt{\alpha_{i}}}{\sqrt{\alpha_{i}}+\sqrt{\alpha_{j}}}\right)^{2}\left\|\operatorname{curl}_{h}^{i}\right\|_{0, \Omega_{i}}^{2} .
\end{aligned}
$$

A similar estimate holds on $\Gamma_{j}$ as well. Substituting these inequalities into (5.22) yields

$$
\begin{aligned}
& \left\|\operatorname{curl}_{i j}^{i}\right\|_{0, \Omega_{i}}^{2}+\left\|\mathbf{w}_{i j}^{i}\right\|_{0, \Omega_{i}}^{2} \\
& \quad \lesssim[1+\log (d / h)]^{2}\left[\left(\frac{\sqrt{\alpha_{i}}}{\sqrt{\alpha_{i}}+\sqrt{\alpha_{j}}}\right)^{2}\left\|\operatorname{curl} \mathbf{v}_{h}^{i}\right\|_{0, \Omega_{i}}^{2}+\left(\frac{\sqrt{\alpha_{j}}}{\sqrt{\alpha_{i}}+\sqrt{\alpha_{j}}}\right)^{2}\left\|\operatorname{curl} \mathbf{v}_{h}^{j}\right\|_{0, \Omega_{j}}^{2}\right] .
\end{aligned}
$$

With this estimate, (5.20) and (5.21), we come to

$$
\begin{aligned}
& \left(A_{i}\left(\mathbf{v}_{i j} \mid \Omega_{i}\right),\left.\mathbf{v}_{i j}\right|_{\Omega_{i}}\right)_{\Omega_{i}} \\
& \lesssim[1+\log (d / h)]^{2} \alpha_{i}\left[\left(\frac{\sqrt{\alpha_{i}}}{\sqrt{\alpha_{i}}+\sqrt{\alpha_{j}}}\right)^{2}\left(\left|p_{h}^{i}\right|_{1, \Omega_{i}}^{2}+\left\|\operatorname{curl} \mathbf{v}_{h}^{i}\right\|_{0, \Omega_{i}}^{2}\right)\right. \\
& \left.\quad+\left(\frac{\sqrt{\alpha_{j}}}{\sqrt{\alpha_{i}}+\sqrt{\alpha_{j}}}\right)^{2}\left(\left|p_{h}^{j}\right|_{1, \Omega_{j}}^{2}+\left\|\operatorname{curl}_{h}^{j}\right\|_{0, \Omega_{j}}^{2}\right)\right] .
\end{aligned}
$$

Similarly, we have

$$
\begin{aligned}
& \left(A_{j}\left(\mathbf{v}_{i j} \mid \Omega_{j}\right), \mathbf{v}_{i j} \mid \Omega_{j}\right) \Omega_{\Omega_{j}} \\
& \lesssim[1+\log (d / h)]^{2} \alpha_{j}\left[\left(\frac{\sqrt{\alpha_{i}}}{\sqrt{\alpha_{i}}+\sqrt{\alpha_{j}}}\right)^{2}\left(\left|p_{h}^{i}\right|_{1, \Omega_{i}}^{2}+\left\|\operatorname{curl} \mathbf{v}_{h}^{i}\right\|_{0, \Omega_{i}}^{2}\right)\right. \\
& \left.\quad+\left(\frac{\sqrt{\alpha_{j}}}{\sqrt{\alpha_{i}}+\sqrt{\alpha_{j}}}\right)^{2}\left(\left|p_{h}^{j}\right|_{1, \Omega_{j}}^{2}+\left\|\operatorname{curl} \mathbf{v}_{h}^{j}\right\|_{0, \Omega_{j}}^{2}\right)\right] .
\end{aligned}
$$

Summing this inequality with (5.24) and noting that

$$
\alpha_{i}+\alpha_{j}<\left(\sqrt{\alpha_{i}}+\sqrt{\alpha_{j}}\right)^{2},
$$

we obtain

$$
\begin{aligned}
\left(\hat{A}_{i j} \mathbf{v}_{i j}, \mathbf{v}_{i j}\right) \lesssim & {[1+\log (d / h)]^{2}\left[\alpha_{i}\left(\left|p_{h}^{i}\right|_{1, \Omega_{i}}^{2}+\left\|\operatorname{curl} \mathbf{v}_{h}^{i}\right\|_{0, \Omega_{i}}^{2}\right)\right.} \\
& \left.+\alpha_{j}\left(\left|p_{h}^{j}\right|_{1, \Omega_{j}}^{2}+\left\|\operatorname{curl}_{h} \mathbf{v}_{h}^{j}\right\|_{0, \Omega_{j}}^{2}\right)\right]
\end{aligned}
$$

or

$$
\sum_{\Gamma_{i j}}\left(\hat{A}_{i j} \mathbf{v}_{i j}, \mathbf{v}_{i j}\right) \lesssim[1+\log (d / h)]^{2} \sum_{i=1}^{N} \alpha_{i}\left(\left|p_{h}^{i}\right|_{1, \Omega_{i}}^{2}+\left\|\operatorname{curl} \mathbf{v}_{h}^{i}\right\|_{0, \Omega_{i}}^{2}\right) .
$$

This, together with (4.17), implies

$$
\begin{aligned}
\sum_{\Gamma_{i j}}\left(\hat{A}_{i j} \mathbf{v}_{i j}, \mathbf{v}_{i j}\right) & \lesssim[1+\log (d / h)]^{2} \sum_{i=1}^{N} \alpha_{i}\left(\left\|\operatorname{curl} \mathbf{v}_{h}^{i}\right\|_{0, \Omega_{i}}^{2}+\left\|\mathbf{v}_{h}^{i}\right\|_{0, \Omega_{i}}^{2}\right) \\
& =[1+\log (d / h)]^{2} \sum_{i=1}^{N}\left(A_{i} \mathbf{v}_{h}^{i}, \mathbf{v}_{h}^{i}\right)_{\Omega_{i}} \\
& =[1+\log (d / h)]^{2}\left(\tilde{\tilde{A}} \mathbf{v}_{h}, \mathbf{v}_{h}\right) .
\end{aligned}
$$

The estimates (5.12) and (5.19) indicate that the constant $C_{1}$ in (5.7) can be bounded by $C[1+\log (d / h)]^{2}$. 
Step 4: Estimate the constant $C_{2}$ in (5.8).

Using the triangle inequality, (4.47) and Lemma 4.9 yields

$$
\begin{aligned}
& \left(\tilde{A}\left(\mathbf{w}_{0}+\sum_{\Gamma_{i j}} \mathbf{w}_{i j}\right), \mathbf{w}_{0}+\sum_{\Gamma_{i j}} \mathbf{w}_{i j}\right) \\
& \lesssim\left(\tilde{A} \mathbf{w}_{0}, \mathbf{w}_{0}\right)+\left(\tilde{A} \sum_{\Gamma_{i j}} \mathbf{w}_{i j}, \sum_{\Gamma_{i j}} \mathbf{w}_{i j}\right) \\
& \lesssim\left(\hat{A}_{0} \mathbf{w}_{0}, \mathbf{w}_{0}\right)+\sum_{k=1}^{N}\left(\left.A_{k}\left(\sum_{\Gamma_{i j}} \mathbf{w}_{i j}\right)\right|_{\Omega_{k}},\left.\left(\sum_{\Gamma_{i j}} \mathbf{w}_{i j}\right)\right|_{\Omega_{k}}\right)_{\Omega_{k}} .
\end{aligned}
$$

It is easy to see that

$$
\begin{aligned}
& \sum_{k=1}^{N}\left(\left.A_{k}\left(\sum_{\Gamma_{i j}} \mathbf{w}_{i j}\right)\right|_{\Omega_{k}},\left.\left(\sum_{\Gamma_{i j}} \mathbf{w}_{i j}\right)\right|_{\Omega_{k}}\right)_{\Omega_{k}} \\
& =\sum_{k=1}^{N} \sum_{\Gamma_{i j}}\left(A_{k}\left(\left.\mathbf{w}_{i j}\right|_{\Omega_{k}}\right), \sum_{\Gamma_{i j}}\left(\left.\mathbf{w}_{i j}\right|_{\Omega_{k}}\right)\right)_{\Omega_{k}} \\
& \lesssim \sum_{k=1}^{N} \sum_{\Gamma_{i j} \subset \Gamma_{k}}\left(A_{k}\left(\mathbf{w}_{i j} \mid \Omega_{k}\right),\left.\mathbf{w}_{i j}\right|_{\Omega_{k}}\right)_{\Omega_{k}} .
\end{aligned}
$$

As each face $\Gamma_{i j}$ is shared by only two subdomains $\Omega_{i}$ and $\Omega_{j}$, we have

$$
\sum_{k=1}^{N}\left(\left.A_{k}\left(\sum_{\Gamma_{i j}} \mathbf{w}_{i j}\right)\right|_{\Omega_{k}},\left.\left(\sum_{\Gamma_{i j}} \mathbf{w}_{i j}\right)\right|_{\Omega_{k}}\right)_{\Omega_{k}} \lesssim \sum_{\Gamma_{i j}}\left(\hat{A}_{i j} \mathbf{w}_{i j}, \mathbf{w}_{i j}\right) .
$$

This, together with (5.26), indicates that the constant $C_{2}$ in (5.8) is bounded by a constant independent of $h$ and $d$.

5.3. Proof of Theorem 3.3. One can verify that (cf. [5])

$$
\left(B^{t} \tilde{A}^{-1} B q, q\right)=\sup _{\mathbf{v} \in V_{h}(\Omega)} \frac{(\mathbf{v}, B q)^{2}}{(\tilde{A} \mathbf{v}, \mathbf{v})}, \quad q \in Z_{h}(\Omega) .
$$

By the definitions of $B$, we have

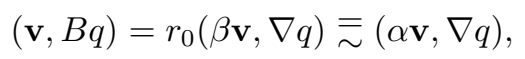

so we have

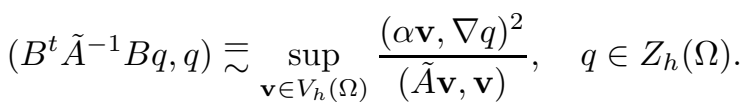

If we choose $\mathbf{v}=\nabla q$, then $(\tilde{A} \mathbf{v}, \mathbf{v})=(\alpha \nabla q, \nabla q)$, and

$$
\left(B^{t} \tilde{A}^{-1} B q, q\right) \geq \frac{(\alpha \nabla q, \nabla q)^{2}}{(\alpha \nabla q, \nabla q)}=\left\|\alpha^{\frac{1}{2}} \nabla q\right\|_{0, \Omega} .
$$

On the other hand, by the Cauchy-Schwarz inequality and the definition of $\tilde{A}$ we obtain

$$
\frac{(\alpha \mathbf{v}, \nabla q)^{2}}{(\tilde{A} \mathbf{v}, \mathbf{v})} \leq \frac{\left\|\alpha^{\frac{1}{2}} \mathbf{v}\right\|_{0, \Omega}^{2} \cdot\left\|\alpha^{\frac{1}{2}} \nabla q\right\|_{0, \Omega}^{2}}{\left\|\alpha^{\frac{1}{2}} \mathbf{v}\right\|_{0, \Omega}^{2}}=\left\|\alpha^{\frac{1}{2}} \nabla q\right\|_{0, \Omega}^{2}, \forall \mathbf{v} \in V_{h}(\Omega)
$$


Thus by (5.27) we have

$$
\left(B^{t} \tilde{A}^{-1} B q, q\right) \lesssim\left\|\alpha^{\frac{1}{2}} \nabla q\right\|_{0, \Omega}^{2} .
$$

This, together with (5.28), leads to

$$
\left(B^{t} \tilde{A}^{-1} B q, q\right) \equiv\left\|\alpha^{\frac{1}{2}} \nabla q\right\|_{0, \Omega}^{2}, \quad \forall q \in Z_{h}(\Omega) .
$$

Therefore, we infer from (3.3) that

$$
\operatorname{cond}\left(\hat{C}^{-1} B^{t} \tilde{A}^{-1} B\right) \lesssim G(d / h),
$$

which, together with Theorem 3.2, yields (3.8).

\section{Appendix}

Lemma 6.1. For any $p_{h}^{i} \in Z_{h}\left(\Omega_{i}\right)$, let $p_{h 0}^{i}=R_{h}^{i} \pi_{0}^{i}\left(\left.p_{h}^{i}\right|_{\Gamma_{i}}\right) \in Z_{h}\left(\Omega_{i}\right)$. We have

$$
\left\|\nabla p_{h 0}^{i} \times \mathbf{n}\right\|_{0, \mathrm{~F}_{b}} \lesssim h^{-\frac{1}{2}}\left\|p_{h}^{i}-\gamma_{\mathrm{F}}\left(p_{h}^{i}\right)\right\|_{0, \partial \mathrm{F}}, \quad \mathrm{F} \subset \Gamma_{i} .
$$

Proof. Let $G$ be a fine triangle in $\mathrm{F}_{b}$, and let $A_{i}(i=1,2,3)$ be the vertices of $G$. Without loss of generality, we assume that $A_{1}, A_{2} \in \partial \mathrm{F}$. Let $K$ be the element of $\Omega_{i}$ which has a face $G$, and let $A_{4} \in \Omega_{i}$ be another vertex of $K$. Let $\lambda_{i}(x, y, z)$ denote the nodal basis function at the node $A_{i}$. Then

$$
p_{h}^{i}(x, y, z)=\sum_{k=1}^{4} p_{h}^{i}\left(A_{k}\right) \lambda_{k}(x, y, x), \quad \forall(x, y, z) \in K .
$$

By the definition of $p_{h 0}^{i}$, we have

$$
p_{h 0}^{i}-\gamma_{\mathrm{F}}\left(p_{h}^{i}\right)=\sum_{k=1}^{2}\left(p_{h}^{i}\left(A_{k}\right)-\gamma_{\mathrm{F}}\left(p_{h}^{i}\right)\right) \lambda_{k}+\hat{a}_{4} \lambda_{4} \quad \text { on } K .
$$

It follows from Lemma 4.16 that $\nabla \lambda_{4} \times \mathbf{n}=0$ on $G$. Thus,

$$
\nabla p_{h 0}^{i} \times\left.\mathbf{n}\right|_{G}=\nabla\left(p_{h 0}^{i}-\gamma_{\mathrm{F}}\left(p_{h}^{i}\right)\right) \times\left.\mathbf{n}\right|_{G}=\sum_{k=1}^{2}\left(p_{h}^{i}\left(A_{k}\right)-\gamma_{\mathrm{F}}\left(p_{h}^{i}\right)\right) \nabla \lambda_{k} \times\left.\mathbf{n}\right|_{G}
$$

It can be verified that

$$
\left\|\nabla \lambda_{k} \times \mathbf{n}\right\|_{0, G} \lesssim 1, \quad k=1,2,
$$

which, together with (6.2), yields

$$
\left\|\nabla p_{h 0}^{i} \times \mathbf{n}\right\|_{0, G} \lesssim \sum_{k=1}^{2}\left|p_{h}^{i}\left(A_{k}\right)-\gamma_{\mathrm{F}}\left(p_{h}^{i}\right)\right| .
$$

Summing this over all $G \subset \mathrm{F}_{b}$ leads to

$$
\left\|\nabla p_{h 0}^{i} \times \mathbf{n}\right\|_{0, \mathrm{~F}_{b}}^{2} \lesssim \sum_{A \in \partial \mathrm{F}}\left|p_{h}^{i}(A)-\gamma_{\mathrm{F}}\left(p_{h}^{i}\right)\right|^{2}
$$

where $A$ denotes a node of the fine mesh on $\partial \mathrm{F}$. Now, (6.1) follows from the equivalence between the $L^{2}$-norm and the discrete $L^{2}$-norm on $\partial \mathrm{F}$. 
Lemma 6.2. Let $\mathbf{v}_{0} \in V^{0}(\Omega)$ be defined as in subsection 5.2. Then we have the identity

$$
\begin{aligned}
& \left.\operatorname{div}_{\tau}\left(\mathbf{v}_{0} \times \mathbf{n}\right)\right|_{\Gamma_{i}}=\left.\operatorname{div}_{\tau}\left(\mathbf{v}_{h}^{i} \times \mathbf{n}\right)\right|_{\Gamma_{i}} \\
& -\sum_{\mathrm{F} \subset \Gamma_{i}}\left[\left.\frac{\sqrt{\alpha_{i}}}{\sqrt{\alpha_{i}}+\sqrt{\alpha_{j}}} \operatorname{div}_{\tau} \mathbf{I}_{\mathrm{F}_{\partial}}^{0}\left(\mathbf{w}_{h}^{i} \times \mathbf{n}\right)\right|_{\Gamma_{i}}+\left.\frac{\sqrt{\alpha_{i}}}{\sqrt{\alpha_{i}}+\sqrt{\alpha_{j}}} \operatorname{div}_{\tau} \mathbf{I}_{\mathrm{F}_{\partial}}^{0}\left(\mathbf{w}_{h}^{j} \times \mathbf{n}\right)\right|_{\Gamma_{j}}\right] .
\end{aligned}
$$

Proof. It is easy to see that

$$
\begin{aligned}
& \nabla p_{h 0}^{i}+\mathbf{w}_{h 0}^{i}=\mathbf{v}_{h}^{i}+\nabla\left(p_{h 0}^{i}-p_{h}^{i}\right)+\mathbf{w}_{h 0}^{i}-\mathbf{w}_{h}^{i}, \\
& \nabla p_{h 0}^{j}+\mathbf{w}_{h 0}^{j}=\mathbf{v}_{h}^{j}+\nabla\left(p_{h 0}^{j}-p_{h}^{j}\right)+\mathbf{w}_{h 0}^{j}-\mathbf{w}_{h}^{j} .
\end{aligned}
$$

Let $\mathrm{F}=\Gamma_{i j}$. Then we have directly by the definition of $\mathbf{v}_{0}$ that

$$
\begin{aligned}
\left.\left(\mathbf{v}_{0} \times \mathbf{n}\right)\right|_{\mathrm{F}} & =\left.\frac{\sqrt{\alpha_{i}}}{\sqrt{\alpha_{i}}+\sqrt{\alpha_{j}}}\left(\mathbf{v}_{h}^{i} \times \mathbf{n}\right)\right|_{\mathrm{F}}+\left.\frac{\sqrt{\alpha_{j}}}{\sqrt{\alpha_{i}}+\sqrt{\alpha_{j}}}\left(\mathbf{v}_{h}^{j} \times \mathbf{n}\right)\right|_{\mathrm{F}} \\
& +\frac{\sqrt{\alpha_{i}}}{\sqrt{\alpha_{i}}+\sqrt{\alpha_{j}}} \nabla\left(p_{h 0}^{i}-p_{h}^{i}\right) \times\left.\mathbf{n}\right|_{\mathrm{F}}+\frac{\sqrt{\alpha_{j}}}{\sqrt{\alpha_{i}}+\sqrt{\alpha_{j}}} \nabla\left(p_{h 0}^{j}-p_{h}^{j}\right) \times\left.\mathbf{n}\right|_{\mathrm{F}} \\
& +\frac{\sqrt{\alpha_{i}}}{\sqrt{\alpha_{i}}+\sqrt{\alpha_{j}}}\left(\mathbf{w}_{h 0}^{i}-\mathbf{w}_{h}^{i}\right) \times\left.\mathbf{n}\right|_{\mathrm{F}}+\frac{\sqrt{\alpha_{j}}}{\sqrt{\alpha_{i}}+\sqrt{\alpha_{j}}}\left(\mathbf{w}_{h 0}^{j}-\mathbf{w}_{h}^{j}\right) \times\left.\mathbf{n}\right|_{\mathrm{F}} .
\end{aligned}
$$

But note that $\mathbf{v}_{h}^{i} \times \mathbf{n}=\mathbf{v}_{h}^{j} \times \mathbf{n}$ on F. Then

$$
\frac{\sqrt{\alpha_{i}}}{\sqrt{\alpha_{i}}+\sqrt{\alpha_{j}}}\left(\mathbf{v}_{h}^{i} \times \mathbf{n}\right)+\frac{\sqrt{\alpha_{j}}}{\sqrt{\alpha_{i}}+\sqrt{\alpha_{j}}}\left(\mathbf{v}_{h}^{j} \times \mathbf{n}\right)=\mathbf{v}_{h}^{i} \times \mathbf{n} \quad \text { on } \mathrm{F} .
$$

This implies

$$
\sum_{\mathrm{F} \subset \Gamma_{i}} \mathbf{I}_{\mathrm{F}}^{t}\left[\left.\frac{\sqrt{\alpha_{i}}}{\sqrt{\alpha_{i}}+\sqrt{\alpha_{j}}}\left(\mathbf{v}_{h}^{i} \times \mathbf{n}\right)\right|_{\mathrm{F}}+\left.\frac{\sqrt{\alpha_{j}}}{\sqrt{\alpha_{i}}+\sqrt{\alpha_{j}}}\left(\mathbf{v}_{h}^{j} \times \mathbf{n}\right)\right|_{\mathrm{F}}\right]=\left.\left(\mathbf{v}_{h}^{i} \times \mathbf{n}\right)\right|_{\Gamma_{i}} .
$$

Again noting the fact that

$$
\begin{array}{ll}
\left(p_{h 0}^{i}-p_{h}^{i}\right)-\left(\left.R_{h}^{i} \mathrm{I}_{\mathrm{F}}^{t}\left(p_{h 0}^{i}-p_{h}^{i}\right)\right|_{\mathrm{F}}\right)=0 & \text { on } \mathrm{F}, \\
\left(p_{h 0}^{j}-p_{h}^{j}\right)-\left(\left.R_{h}^{i} \mathrm{I}_{\mathrm{F}}^{t}\left(p_{h 0}^{j}-p_{h}^{j}\right)\right|_{\mathrm{F}}\right)=0 & \text { on } \mathrm{F},
\end{array}
$$

we derive by Lemma 6.1 that

$$
\begin{aligned}
\frac{\sqrt{\alpha_{i}}}{\sqrt{\alpha_{i}}+\sqrt{\alpha_{j}}} \nabla\left(p_{h 0}^{i}-p_{h}^{i}\right) \times\left.\mathbf{n}\right|_{\mathrm{F}}+\frac{\sqrt{\alpha_{j}}}{\sqrt{\alpha_{i}}+\sqrt{\alpha_{j}}} \nabla\left(p_{h 0}^{j}-p_{h}^{j}\right) \times\left.\mathbf{n}\right|_{\mathrm{F}} \\
=\frac{\sqrt{\alpha_{i}}}{\sqrt{\alpha_{i}}+\sqrt{\alpha_{j}}}\left(\left.\nabla R_{h}^{i} \mathrm{I}_{\mathrm{F}}^{t}\left(p_{h 0}^{i}-p_{h}^{i}\right)\right|_{\mathrm{F}}\right) \\
\quad \times\left.\mathbf{n}\right|_{\mathrm{F}}+\frac{\sqrt{\alpha_{j}}}{\sqrt{\alpha_{i}}+\sqrt{\alpha_{j}}}\left(\left.\nabla R_{h}^{i} \mathrm{I}_{\mathrm{F}}^{t}\left(p_{h 0}^{j}-p_{h}^{j}\right)\right|_{\mathrm{F}}\right) \times\left.\mathbf{n}\right|_{\mathrm{F}} \\
=\left[\nabla R_{h}^{i} \mathrm{I}_{\mathrm{F}}^{t}\left(\left.\frac{\sqrt{\alpha_{i}}}{\sqrt{\alpha_{i}}+\sqrt{\alpha_{j}}}\left(p_{h 0}^{i}-p_{h}^{i}\right)\right|_{\mathrm{F}}+\left.\frac{\sqrt{\alpha_{j}}}{\sqrt{\alpha_{i}}+\sqrt{\alpha_{j}}}\left(p_{h 0}^{j}-p_{h}^{j}\right)\right|_{\mathrm{F}}\right)\right] \times\left.\mathbf{n}\right|_{\mathrm{F}} .
\end{aligned}
$$

Hence,

$$
\begin{aligned}
& \sum_{\mathrm{F} \subset \Gamma_{i}} \mathbf{I}_{\mathrm{F}}^{t}\left[\frac{\sqrt{\alpha_{i}}}{\sqrt{\alpha_{i}}+\sqrt{\alpha_{j}}} \nabla\left(p_{h 0}^{i}-p_{h}^{i}\right) \times\left.\mathbf{n}\right|_{\mathrm{F}}+\frac{\sqrt{\alpha_{j}}}{\sqrt{\alpha_{i}}+\sqrt{\alpha_{j}}} \nabla\left(p_{h 0}^{j}-p_{h}^{j}\right) \times\left.\mathbf{n}\right|_{\mathrm{F}}\right] \\
& =\left[\nabla R_{h}^{i} \sum_{\mathrm{F} \subset \Gamma_{i}} \mathrm{I}_{\mathrm{F}}^{t}\left(\left.\frac{\sqrt{\alpha_{i}}}{\sqrt{\alpha_{i}}+\sqrt{\alpha_{j}}}\left(p_{h 0}^{i}-p_{h}^{i}\right)\right|_{\mathrm{F}}+\left.\frac{\sqrt{\alpha_{j}}}{\sqrt{\alpha_{i}}+\sqrt{\alpha_{j}}}\left(p_{h 0}^{j}-p_{h}^{j}\right)\right|_{\mathrm{F}}\right)\right] \times\left.\mathbf{n}\right|_{\Gamma_{i}} .
\end{aligned}
$$


In addition, it is easy to see that

$$
\begin{aligned}
\sum_{\mathrm{F} \subset \Gamma_{i}} & \mathbf{I}_{\mathrm{F}}^{t}\left[\frac{\sqrt{\alpha_{i}}}{\sqrt{\alpha_{i}}+\sqrt{\alpha_{j}}}\left(\mathbf{w}_{h 0}^{i}-\mathbf{w}_{h}^{i}\right) \times\left.\mathbf{n}\right|_{\mathrm{F}}+\frac{\sqrt{\alpha_{j}}}{\sqrt{\alpha_{i}}+\sqrt{\alpha_{j}}}\left(\mathbf{w}_{h 0}^{j}-\mathbf{w}_{h}^{j}\right) \times\left.\mathbf{n}\right|_{\mathrm{F}}\right] \\
& =-\sum_{\mathrm{F} \subset \Gamma_{i}}\left[\left.\frac{\sqrt{\alpha_{i}}}{\sqrt{\alpha_{i}}+\sqrt{\alpha_{j}}} \mathbf{I}_{\mathrm{F}_{\partial}}^{0}\left(\mathbf{w}_{h}^{i} \times \mathbf{n}\right)\right|_{\Gamma_{i}}+\left.\frac{\sqrt{\alpha_{j}}}{\sqrt{\alpha_{i}}+\sqrt{\alpha_{j}}} \mathbf{I}_{\mathrm{F}_{\partial}}^{0}\left(\mathbf{w}_{h}^{j} \times \mathbf{n}\right)\right|_{\Gamma_{j}}\right] .
\end{aligned}
$$

Summing (6.4) over $\mathrm{F} \subset \Gamma_{i}$ and using (6.5)-(6.7) yield

$$
\begin{aligned}
& \left.\left(\mathbf{v}_{0} \times \mathbf{n}\right)\right|_{\Gamma_{i}}=\left.\sum_{\mathrm{F} \subset \Gamma_{i}} \mathbf{I}_{\mathrm{F}}^{t}\left(\mathbf{v}_{0} \times \mathbf{n}\right)\right|_{\mathrm{F}}=\left.\left(\mathbf{v}_{h}^{i} \times \mathbf{n}\right)\right|_{\Gamma_{i}} \\
& +\left[\nabla R_{h}^{i} \sum_{\mathrm{F} \subset \Gamma_{i}} \mathrm{I}_{\mathrm{F}}^{t}\left(\left.\frac{\sqrt{\alpha_{i}}}{\sqrt{\alpha_{i}}+\sqrt{\alpha_{j}}}\left(p_{h 0}^{i}-p_{h}^{i}\right)\right|_{\mathrm{F}}+\left.\frac{\sqrt{\alpha_{j}}}{\sqrt{\alpha_{i}}+\sqrt{\alpha_{j}}}\left(p_{h 0}^{j}-p_{h}^{j}\right)\right|_{\mathrm{F}}\right)\right] \times\left.\mathbf{n}\right|_{\Gamma_{i}} \\
& -\sum_{\mathrm{F} \subset \Gamma_{i}}\left[\left.\frac{\sqrt{\alpha_{i}}}{\sqrt{\alpha_{i}}+\sqrt{\alpha_{j}}} \mathbf{I}_{\mathrm{F}_{\partial}}^{0}\left(\mathbf{w}_{h}^{i} \times \mathbf{n}\right)\right|_{\Gamma_{i}}+\left.\frac{\sqrt{\alpha_{j}}}{\sqrt{\alpha_{i}}+\sqrt{\alpha_{j}}} \mathbf{I}_{\mathrm{F}_{\partial}}^{0}\left(\mathbf{w}_{h}^{j} \times \mathbf{n}\right)\right|_{\Gamma_{j}}\right] .
\end{aligned}
$$

Now (6.3) follows from the fact that $\left.\operatorname{div}_{\tau}\left(\nabla q_{h} \times \mathbf{n}\right)\right|_{\Gamma_{i}}=0$ for any $q_{h} \in Z_{h}\left(\Omega_{i}\right)$.

\section{ACKNOWLEDGMENTS}

The authors wish to thank one anonymous referee for many constructive comments which led to a great improvement of the paper.

\section{REFERENCES}

1. A. Alonso and A. Valli, Some remarks on the characterization of the space of tangential traces of $H(\mathrm{curl} ; \Omega)$ and the construction of an extension operator, Manuscr. Math., 89(1986), 159178. MR 96k:46057

2. A. Alonso and A. Valli, An optimal domain decomposition preconditioner for low-frequency time-harmonic Maxwell equations, Math. Comp., 68(1999), 607-631. MR 99i:78002

3. C. Amrouche, C. Bernardi, M. Dauge and V. Girault. Vector Potentials in three dimensional nonsmooth domains, Math. Meth. Appl. Sci., 21(1998), 823-864. MR 99e:35037

4. J. Bramble, J. Pasciak and A. Schatz, The construction of preconditioner for elliptic problems by substructuring, IV. Math. Comp., 53(1989), 1-24. MR 89m:65098

5. J. Bramble, J. Pasciak and A. Vassilev, Analysis of the inexact Uzawa algorithm for saddlepoint problems, SIAM J. Numer. Anal., 34(1997), 1072-1092. MR 98c:65182

6. J. Bramble and J. Pasciak, A preconditioning technique for indefinite systems resulting from mixed approximations of elliptic problems, Math. Comp., 50(1988), 1-18. MR 89m:65097a

7. M. Cessenat, Mathematical methods in electromagnetism, World Scientific, River Edge, NJ, 1998. MR 97j:78001

8. Z. Chen, Q. Du and J. Zou, Finite element methods with matching and nonmatching meshes for Maxwell equations with discontinuous coefficients, SIAM J. Numer. Anal., 37(1999), 15421570. MR 2001h:78044

9. P. Ciarlet, Jr. and J. Zou, Fully discrete finite element approaches for time-dependent Maxwell's equations, Numer. Math., 82(1999), 193-219 MR 2000c:65083

10. M. Costabel, A remark on the regularity of solution of Maxwell's equations on Lipschitz domains, Math. Meth. Appl. Sci. 12 (1990), 365-368. MR 91c:35028

11. R. Dautray and J.-L. Lions, Mathematical analysis and numerical methods for science and technology, Springer-Verlag, New York, 1988. MR 89m:00001

12. V. Girault and P. Raviart, Finite Element Methods for Navier-Stokes Equations, SpringerVerlag, Berlin, 1986. MR 88b:65129

13. J. Gopalakrishnan and J. Pasciak, Overlapping Schwarz preconditioners for indefinite time harmonic Maxwell's equations, Math. Comp., 72 (2003), 1-16.

14. R. Hiptmair, Multigrid method for Maxwell's equations, SIAM J. Numer. Anal., 36(1998), 204-225. MR 99j:65229 
15. Q. Hu and G. Liang, A general framework to construct interface preconditioners, Chinese J. Num. Math. \& Appl., 21(1999), 83-95. MR 99m:65238

16. Q. Hu, G. Liang and J. Lui, Construction of a preconditioner for three dimensional domain decomposition methods with Lagrangian multipliers, J. Comput. Math., 19 (2001), 213-224. MR 2001k:65186

17. Q. Hu and J. Zou, An iterative method with variable parameters for saddle-point problems, SIAM J. Matrix Anal. Appl., 23(2001), 317-338. MR 2002j:65041

18. Q. Hu and J. Zou, Two new variants of nonlinear inexact Uzawa algorithms for saddle-point problems, Numer. Math., 93 (2002), 333-359.

19. Q. Hu and J. Zou, Substructuring preconditioners for saddle-point problems arising from Maxwell's equations in three dimensions. Technical Report CUHK 2002-16(256), Department of Mathematics, The Chinese University of Hong Kong, 2002.

20. Q. Hu and J. Zou, A non-overlapping domain decomposition method for Maxwell's equations in three dimensions. Accepted for publication in SIAM J. Numer. Anal.

21. P. Monk, Analysis of a finite element method for Maxwell's equations, SIAM J. Numer. Anal., 29(1992), 32-56. MR 93k:65096

22. J. Nédélec, Mixed finite elements in $R^{3}$, Numer. Math., 35(1980), 315-341. MR 81k:65125

23. R. Nicolaides and D. Wang, Convergence analysis of a covolume scheme for Maxwell's equations in three dimensions, Math. Comp., 67(1998), 947-963. MR 98j:65080

24. T. Rusten and R. Winther, A preconditioned iterative method for saddlepoint problems, SIAM J. Matrix Anal. Appl., 13(1992), 887-904. MR 93a:65043

25. J. Saranen, On electric and magnetic problems for vector fields in anisotropic nonhomogeneous media, J. Math. Anal. Appl. 91(1983), 254-275. MR 85i:78004

26. B. Smith, A domain decomposition algorithm for elliptic problems in three dimensions, $\mathrm{Nu}-$ mer. Math., 60(1991), 219-234. MR 92m:65159

27. B. Smith, P. Bjorstad and W. Gropp, Domain Decomposition: Parallel multilevel methods for elliptic partial differential equations, Cambridge University Press, 1996. MR 98g:65003

28. P. Tallec, Domain Decomposition Methods in Computational Mechanics, Comput. Mech. Adv., 2: 1321-220, 1994.

29. A. Toselli, Overlapping Schwarz methods for Maxwell's equations in three dimensions, Numer. Math., 86(2000), 733-752. MR 2001h:65137

30. A. Toselli, O. Widlund and B. Wohlmuth, An iterative substructuring method for Maxwell's equations in two dimensions, Math. Comp. 70 (2001), 935-947. MR 2001j:65140

31. J. Xu, Iterative methods by space decomposition and subspace correction, SIAM Review, 34(1992), 581-613. MR 93k:65029

32. J. Xu and J. Zou, Some nonoverlapping domain decomposition methods, SIAM Review, 24(1998), 857-914. MR 99m:65241

Institute of Computational Mathematics and Scientific/Engineering Computing, Academy of Mathematics and System Sciences, Chinese Academy of Sciences, Beijing 100080, CHINA

E-mail address: hqy@lsec.cc.ac.cn

Department of Mathematics, The Chinese University of Hong Kong, Shatin, N.T., HONG KONG

E-mail address: zou@math.cuhk.edu.hk 